\title{
Towards a cosmological neutrino mass detection
}

\author{
R. Allison, ${ }^{1}$ P. Caucal, ${ }^{2,1}$ E. Calabrese, ${ }^{1,3}$ J. Dunkley, ${ }^{1}$ and T. Louis ${ }^{1}$ \\ ${ }^{1}$ Subdepartment of Astrophysics, University of Oxford, Denys Wilkinson Building, \\ Oxford OX1 3RH, United Kingdom \\ ${ }^{2}$ Master ICFP, Département de Physique, École Normale Supérieure, 24 rue Lhomond, \\ 75005 Paris, France \\ ${ }^{3}$ Department of Astrophysical Sciences, Peyton Hall, Princeton University, Princeton, \\ New Jersey 08544, USA
}

(Received 24 September 2015; published 23 December 2015)

\begin{abstract}
Future cosmological measurements should enable the sum of neutrino masses to be determined indirectly through their effects on the expansion rate of the Universe and the clustering of matter. We consider prospects for the gravitationally lensed cosmic microwave background (CMB) anisotropies and baryon acoustic oscillations (BAOs) in the galaxy distribution, examining how the projected uncertainty of $\approx 15 \mathrm{meV}$ on the neutrino mass sum (a $4 \sigma$ detection of the minimal mass) might be reached over the next decade. The current $1 \sigma$ uncertainty of $\approx 103 \mathrm{meV}$ (Planck-2015 + BAO-15) will be improved by upcoming "Stage-3" (S3) CMB experiments (S3+BAO-15:44 meV), then upcoming BAO measurements (S3 + DESI: $22 \mathrm{meV})$, and planned next-generation "Stage 4" (S4) CMB experiments (S4 + DESI: 15-19 meV, depending on angular range). An improved optical depth measurement is important: the projected neutrino mass uncertainty increases to $26 \mathrm{meV}$ if $\mathrm{S} 4$ is limited to $\ell>20$ and combined with current large-scale polarization data. Looking beyond $\Lambda \mathrm{CDM}$, including curvature uncertainty increases the forecast mass error by $\approx 50 \%$ for S4 + DESI, and more than doubles the error with a two-parameter dark-energy equation of state. Complementary low-redshift probes including galaxy lensing will play a role in distinguishing between massive neutrinos and a departure from a $w=-1$, flat geometry.
\end{abstract}

DOI: 10.1103/PhysRevD.92.123535

PACS numbers: 98.80.-k, 14.60.Pq, 95.85.Ry

\section{INTRODUCTION}

A central goal in both cosmology and particle physics is to measure the mass of the neutrino particles. The neutrino sector is still poorly understood and the mechanism that gives rise to their mass is unknown. There are thought to be three active neutrino species, with mass differences measured through solar, atmospheric, reactor, and accelerator neutrino oscillation experiments (for reviews see, e.g., Gonzalez-Garcia and Nir [1], Maltoni et al. [2], Smirnov [3], and Feldman et al. [4]). The results imply a minimum total mass of $60 \mathrm{meV}$ in a normal hierarchy with two lighter neutrinos and one heavier neutrino, or $100 \mathrm{meV}$ in an inverted hierarchy with two massive neutrinos.

Cosmology provides an indirect probe of massive neutrinos (e.g., [5-19]). Massive neutrinos behave initially like noninteracting relativistic particles, and then later like cold dark matter. As such, they affect the expansion rate of the Universe, compared to a pure radiation or pure matter component, and they modify the evolution of perturbations at early times. They also modify the growth of structure through a suppression of the clustering of matter on scales that entered the cosmic horizon while the neutrinos were relativistic.

The current indirect 95\% upper limit from cosmological data on the sum of the neutrino masses is $\Sigma m_{\nu}<230 \mathrm{meV}$ from the Planck measurements of the cosmic microwave background (CMB), combined with baryon acoustic oscillation (BAO) measurements from the Baryon Oscillation Spectroscopic Survey (BOSS) [20,21]. The limit is $\Sigma m_{\nu}<$ $680 \mathrm{meV}$ from the CMB alone [21]. Tighter limits have been found including Lyman- $\alpha$ forest measurements from quasars in the BOSS survey $\left(\Sigma m_{\nu}<120 \mathrm{meV}\right)[22,23]$, but the result depends on numerical hydrodynamical simulations which may contribute additional systematic uncertainty.

Recent forecasts of mass limits for upcoming cosmological data sets, including galaxy lensing and clustering, redshift-space distortions, the kinematic SunyaevZel'dovich effect, and counts of galaxy clusters, have been studied extensively (e.g., Kitching et al. [24], Font-Ribera et al. [25], Villaescusa-Navarro et al. [26], Mueller et al. [27], and Errard et al. [28]), showing the promise of a wide range of future cosmological data to target a neutrino mass measurement. In this paper we focus on the combination of lensed $\mathrm{CMB}$ and $\mathrm{BAO}$ measurements, data sets which do not require detailed modeling of nonlinear structure formation or an understanding of galaxy bias. The gravitationally lensed CMB measures the growth of structure at times typically before the Universe was half its current age, and on angular scales larger than $\approx 100 \mathrm{Mpc}$, and so it is dominated by linear physics. Studies of this combination have been reported in Hall and Challinor [29], Abazajian et al. [30], Wu et al. [31], and Pan and Knox [32], with a $4 \sigma$ 
detection of neutrino mass forecast for the next generation of experiments. In this paper we investigate this further, exploring the dependence on experimental details and on parameter degeneracies.

In Sec. II we give a brief review of the cosmological effects of neutrinos, and in Sec. III we study how the mass measurement may be reached stepwise using data collected during the coming decade. In Sec. IV we investigate the dependence on experimental details, and in Sec. V we explore degeneracies with other cosmological parameters. We conclude in Sec. VI.

\section{COSMOLOGICAL EFFECTS OF NEUTRINOS}

Standard Model neutrinos are initially relativistic, following a thermal distribution after decoupling from the primordial plasma when the Universe had a temperature of around $k_{B} T \approx 1 \mathrm{MeV}$. The neutrino temperature decreases as the scale factor grows, until their rest-mass energy dominates and they become indistinguishable from cold dark matter. For a neutrino of mass $m_{\nu}$ the transition occurs at $z \approx 120\left(\frac{m_{\nu}}{60 \mathrm{meV}}\right)$ [12], so current limits indicate a transition epoch of $120 \lesssim z \lesssim 460$ for a normal mass hierarchy.

This limit implies that the neutrinos were still relativistic when the CMB decoupled, so they would be indistinguishable from massless neutrinos in the primary anisotropies. However, higher mass neutrinos become nonrelativistic sooner, which reduces the early-time integrated SachsWolfe (ISW) effect. This gravitational redshift of the CMB photons arises while the non-negligible radiation component causes the potentials of the density fluctuations to evolve $[7,12]$ and affects the anisotropies on scales around the first acoustic peak [33].

This effect is not sensitive to masses that remain relativistic until well after decoupling [12,34], but further information comes from probes of later-time large-scale structure measurements. Massive neutrinos interact weakly, allowing them to free stream out of overdensities while relativistic, so the growth rate of matter perturbations inside the horizon is suppressed compared to a universe with only cold dark matter. For comoving wave numbers $k \gg k_{\mathrm{FS}}, \mathrm{Hu}$ et al. [35] show that the suppression of the matter power spectrum today, $P(k)$, is proportional to the sum of the neutrino masses,

$$
\frac{P_{\sum m_{\nu}}(k)-P_{\Sigma m_{\nu}=0}(k)}{P_{\Sigma m_{\nu}=0}(k)} \approx-0.07\left(\frac{\sum m_{\nu}}{0.1 \mathrm{eV}}\right)\left(\frac{\Omega_{m} h^{2}}{0.136}\right)^{-1},
$$

where the comoving free-streaming scale is given by

$$
k_{\mathrm{FS}}=0.0072\left(\frac{\Sigma m_{\nu}}{0.1 \mathrm{eV}}\right)^{1 / 2}\left(\frac{\Omega_{m}}{0.315}\right)^{1 / 2} h \mathrm{Mpc}^{-1},
$$

as illustrated in Fig. 1 and, e.g., [30], for models with fixed total matter density. For current limits this scale is estimated to lie in the range $0.005 \lesssim k_{\mathrm{FS}} \lesssim 0.011\left[h \mathrm{Mpc}^{-1}\right]$.
The suppression of small-scale power can be probed using galaxy clustering and the gravitational lensing of galaxies. These are promising avenues for neutrino mass measurements (e.g., [25]), although these observables are sensitive to nonlinearities in the matter power spectrum and scale-dependent galaxy and shape biases [36,37]. An alternative route is through the gravitational lensing of the CMB (see, e.g., [38] for a review). Here the CMB photons are deflected by the large-scale structure, integrated over the photon path after decoupling.

Following [38], the CMB convergence angular power spectrum, $C_{l}^{\kappa \kappa}$, is a weighted projection of the matter power spectrum $P(k, \chi)$; under the Limber approximation,

$$
C_{\ell}^{\kappa \kappa}=\int_{0}^{\chi_{H}} d \chi \frac{W^{2}(\chi)}{f_{k}^{2}(\chi)} P\left(\frac{\ell}{f_{k}(\chi)}, \chi\right),
$$

where $\chi_{H}$ is the comoving horizon size, $f_{k}(\chi)$ relates lineof-sight comoving distances and transverse comoving distances in a curved universe, and the window function $W(\chi)$ is

$$
W(\chi)=\frac{3 \Omega_{m} H_{0}^{2}}{2 c^{2}} \frac{f_{k}(\chi) f_{k}\left(\chi^{*}-\chi\right)}{a(\chi) f_{k}\left(\chi^{*}\right)}
$$

for $\chi<\chi^{*}$ and zero otherwise. Here $a(\chi)$ is the scale factor and $\chi^{*}$ is the radial comoving distance to the last-scattering surface. This angular power spectrum is sensitive to $\Sigma m_{\nu}$, as shown in Fig. 1, and does not depend on galaxy bias. CMB lensing probes structures at higher redshift than galaxy clustering or weak lensing, and is correspondingly less sensitive to detailed nonlinear modeling of the matter power spectrum. In practice it is reconstructed from CMB temperature and polarization maps using a four-point function (e.g., [39]).

The CMB temperature and polarization angular power spectra, $\left\{C_{\ell}^{T T}, C_{\ell}^{T E}, C_{\ell}^{E E}, C_{\ell}^{B B}\right\}$, are also modified by lensing, which smears the acoustic peaks by adding variance to the apparent scale of a mode, converts $E$-mode polarization into $B$-mode polarization, and adds small-scale power in $T$, $E$, and $B$ (e.g., [38]). The approximate effect of massive neutrinos is shown in Fig. 1 for the $E$-mode polarization, where we artificially amplify the effects of neutrinos on the $\mathrm{CMB}$ lensing, rather than the primary $\mathrm{CMB}$, by varying the amplitude of the lensing potential. Increasing the neutrino mass has a similar effect to decreasing the lensing amplitude. Compared to the power spectra, the reconstructed convergence field contains more information on the neutrino mass [40].

Massive neutrinos also affect angular diameter distances $d_{A}(z)$ and the expansion rate $H(z)$, as their evolution differs from a pure radiation or pure matter component (e.g., [32]). These can be measured using a "standard ruler" method that is relatively free of systematic uncertainties: The primordial oscillations in the photon-baryon fluid are imprinted in the 

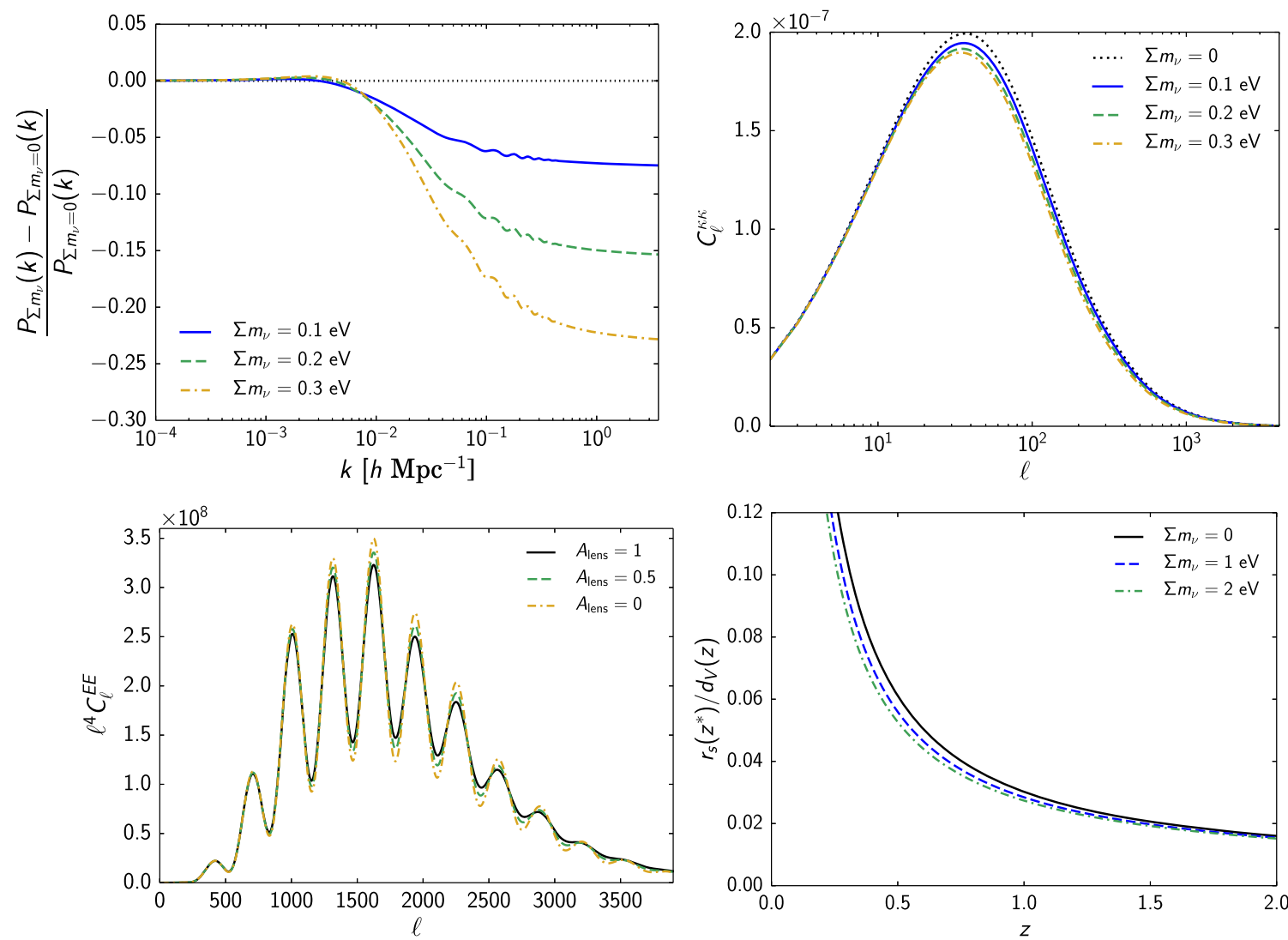

FIG. 1 (color online). Effect of neutrino mass on CMB power spectra and BAO distance scales. Top: Fractional change of the linear matter power spectrum today $P(k)$ (left) and CMB convergence power spectrum $C_{l}^{\kappa \kappa}$ (right) with neutrino mass $\Sigma m_{\nu}$, for fixed physical dark-matter density today, $\Omega_{\mathrm{c}} h^{2}+\Omega_{\nu} h^{2}$. Suppression of power is due to neutrino free-streaming, and the oscillatory structure comes from a slight shift in the acoustic scale between models. Bottom left: Lensed CMB $E$-mode power spectrum with varying amplitudes of the lensing potential $A_{\text {lens }}$, approximating and exaggerating the effect that massive neutrinos have on the CMB polarization spectrum. Bottom right: BAO distance ratio $r_{s} / d_{V}$ for fixed $\theta_{A}$ and $\Omega_{c} h^{2}$. Massive neutrinos behave like additional matter in the BAO redshift range, decreasing $H_{0}$ and increasing the volume distance $d_{V}$.

galaxy distribution as BAOs. The comoving scale of the oscillations is fixed by the sound horizon at decoupling, $r_{s}$, which is not significantly affected by neutrino masses given current limits and is in the linear regime of density perturbations $(\approx 150 \mathrm{Mpc})$. The observed spherically averaged BAO angular scale for galaxies at redshift $z$ is sensitive to the parameter combination $r_{s} / d_{V}(z) ; d_{V}$ is the volume distance [41],

$$
d_{V}(z) \equiv\left[c z(1+z)^{2} d_{A}^{2}(z) H^{-1}(z)\right]^{1 / 3} .
$$

For fixed cold-dark-matter density, more massive neutrinos increase the total late-time nonrelativistic matter content, which increases the volume distance, as shown in Fig 1.

\section{IMPROVEMENTS IN THE NEXT DECADE}

We consider how upcoming and planned $\mathrm{CMB}$ and $\mathrm{BAO}$ experiments will improve the current limits on the sum of the neutrino masses, building on previous analyses [25,29-32].

\section{A. New data}

The current state of the art for the CMB is the Planck and WMAP satellite data, including the first analysis of the full-mission Planck data [21,42]. Improved small-scale CMB measurements are currently being made by the "Stage 2" ground-based experiments: ACTPol, SPTPol, and POLARBEAR [43-45]. These will soon be upgraded to "Stage 3" (hereafter, S3) with new detectors and sensitivity to multiple frequencies. Here we consider a S3 "wide" experiment that maps $40 \%$ of the sky, and a "deep" experiment that maps $6 \%$ of the sky. The wide experiment is similar to AdvACT specifications [46] and the deep to SPT-3G [47]. These experiments are expected to take data from 2016 to 2019, and the specifications we adopt are given in Table I. We also anticipate data from S3 experiments targeting larger angular scales (e.g., CLASS), but do not consider these specifically. In addition, we can expect a complete analysis of the Planck polarization data, optimistically including reliable large-scale polarization data. 
TABLE I. Upcoming (S3) and proposed (S4, CV-low) CMB experiments that will add to Planck. The Planck specifications we use are in the Appendix.

\begin{tabular}{llccc}
\hline \hline Experiment & $f_{\text {sky }}$ & $\begin{array}{c}\text { Beam } \\
(\operatorname{arcmin})\end{array}$ & $\begin{array}{c}\Delta T \\
(\mu \mathrm{K} \text {-arcmin })\end{array}$ & $\begin{array}{c}\Delta P \\
(\mu \mathrm{K} \text {-arcmin })\end{array}$ \\
\hline S3-wide & 0.4 & 1.4 & 8.0 & 11.3 \\
S3-deep & 0.06 & 1 & 4.0 & 5.7 \\
S4 & 0.4 & 3 & 1 & 1.4 \\
CV-low & 0.4 & 60 & 1 & 1.4 \\
\hline \hline
\end{tabular}

Beyond S3, a "Stage-4" (S4) experiment-or set of experiments-is being developed by the CMB community that may cover at least half the sky to typical noise levels of $1 \mu \mathrm{K}$-arcmin [30]. There are also proposed space-based experiments including LiteBIRD and PIXIE $[48,49]$, and we approximate their role with a cosmic variance-limited large-scale polarization measurement ("CV-low") that could supplement S3 ground-based data.

On the BAO front, current state-of-the-art measurements come from the BOSS "LowZ" and "C-MASS" galaxy samples at $z=0.32$ and $z=0.57$ [20]. These are supplemented by data from the Six-Degree Field Galaxy Redshift Survey (6dFGRS) at $z=0.11$ [50] and the Sloan Digital Sky Survey (SDSS) Main Galaxy Sample (MGS) sample at $z=0.15$ [51]. Improved measurements are being made by the eBOSS survey which will survey a deeper sample [52]. A significant advance should be made with the Dark Energy Spectroscopic Instrument (DESI), due to begin surveying in 2018, which is expected to measure the BAO distance ratio from redshifts $0.15<z<1.85$ in bins of width $\Delta z=0.1$ to percent-level precision $[25,53]$.

\section{B. Forecasting methods}

We use a Fisher-matrix forecasting method to predict the neutrino mass uncertainties. For a model defined by parameters $\boldsymbol{\theta}$ the expected Fisher matrix is

$$
F_{i j}(\boldsymbol{\theta})=\left\langle-\frac{\partial^{2} \ln p(\boldsymbol{\theta} \mid d)}{\partial \theta_{i} \partial \theta_{j}}\right\rangle,
$$

where $p(\boldsymbol{\theta} \mid \mathbf{d})$ is the posterior distribution for $\boldsymbol{\theta}$ given data $\mathbf{d}$. The forecast parameter covariance is then given by the inverse of the Fisher matrix, $\mathbb{C}_{i j}=\left(F^{-1}\right)_{i j}$. Here our data are the lensed $T T, T E$, and $E E \mathrm{CMB}$ power spectra, reconstructed $\mathrm{CMB}$ convergence power spectrum $\kappa \kappa$, and BAO distance ratio measurements $r_{s} / d_{V}(z)$. Our parameters are the standard six $\Lambda$ CDM parameters (the physical baryon and cold-dark-matter densities $\Omega_{b} h^{2}$ and $\Omega_{c} h^{2}$, the angular size of the sound horizon at recombination $\theta_{A}$, the optical depth to reionization $\tau$, the amplitude of primordial scalar perturbations $A_{s}$, the tilt of the primordial scalar power $n_{s}$ ) plus the neutrino mass sum $\Sigma m_{\nu}$, as well as possible extension parameters including curvature and dark energy. Our methods are summarized in the Appendix, including choices made about the fiducial model, choice of parameter basis, and step size for calculating derivatives. We also describe validation of our numerical code.

We use the lensed CMB power spectra and the convergence power spectrum as our CMB observables, which differs from the approach in [25,29-31], but more closely follows the "real" data analysis: the CMB sky we see is lensed, and it is a difficult inverse problem to infer the unlensed sky (e.g., [54]). Using unlensed spectra in forecasts removes information contained in the lensed temperature and polarization fields. However, it is challenging to construct the full covariance matrix for the lensed power spectra and convergence power spectrum: the $T, Q$, and $U$ fields are all lensed by the same lensing potential, which correlates the power spectra and adds additional nonGaussian covariance. This is explored in detail in [40,55]. In this analysis we make the approximation of discarding $B B$ information and assuming Gaussian uncertainties in $T T, T E, E E$, and $\kappa \kappa$. We account for the correlation between the temperature and polarization signals and the unlensed $T-\kappa$ correlation (from the ISW effect), but neglect other correlations with $\kappa$. This is likely a good approximation for S3 data, but could underestimate certain parameter errors for S4-type data by up to $\approx 20 \%$ [40]. We use CAMB for evaluation of all relevant CMB and lensing power spectra [56].

For the noise levels of Planck, we consider two cases: "Planck-2015" (P15), which produces cosmological constraints which closely match the published results [21], and "Planck-pol," which includes $T E$ and $E E$ data coming from the polarization measurements of the High Frequency Instrument (HFI), including large scales. The specifications are given in the Appendix. For Planck-pol, noise levels are approximated by taking temperature sensitivities from P15 and assuming the per-channel noise scalings from temperature to polarization in the Planck Blue Book [57]. This is likely to be overoptimistic at the largest scales.

For the CMB power spectra, we set a maximum multipole for the recoverable information: $\ell_{\max }^{T}=3000$, $\ell_{\max }^{P}=4000$, and $\ell_{\max }^{\kappa}=3000$ for the future S3 and S4 experiments. Smaller scales are likely hard to extract due to extragalactic foreground contamination. We assume white noise, and do not include additional foreground uncertainty beyond the multipole cuts outlined above, although the expected S3 white-noise level includes some foreground inflation [46]. We also set a minimum multipole of $\ell_{\min }=$ 50 for S3 due to the challenge of recovering large scales from the ground, and consider two options for $\mathrm{S} 4: \ell_{\min }=$ 50 and $\ell_{\min }=5$. For S3 and S4 we include Planck data for $2<\ell<\ell_{\min }$, and our nominal analysis uses Planck-pol unless stated otherwise. We consider the importance of the large-scale polarization measurements in Sec. IVA.

We use the quadratic-estimator formalism of $\mathrm{Hu}$ and Okamoto [58] to calculate the CMB convergence noise spectrum $N_{\ell}^{\kappa \kappa}$. This uses the coupling of otherwise 
uncorrelated modes in temperature and polarization to reconstruct the lensing potential. Iterative delensing procedures are able to reduce the effective noise level of the estimated lensing field, particularly for the low-noise $(\Delta P \lesssim 4 \mu \mathrm{K}$-arcmin) future experiments considered here [59]. We consider the impact of this process in Sec. III C.

For the BAO measurements we use the published uncertainties on the distance ratio $r_{s} / d_{V}$ for the current BAO data described in Sec. III [20,50,51], labeled as BAO15. For DESI we use the forecast uncertainties on $d_{A}(z)$ and $H(z)$ given in Font-Ribera et al. [25] to estimate the expected $r_{s} / d_{V}$ uncertainties, summarized in the Appendix. We do not use broadband shape information in the galaxy power spectra.

In Fig. 2 we show the fractional changes in lensing and $E$-mode power spectrum and the BAO distance ratio for physically relevant variations in the neutrino mass. We overplot projected uncertainties for S3, S4, BAO-15, and DESI to illustrate the constraining power of these experiments.

\section{Expected constraints}

We find forecast marginal $1 \sigma$ uncertainties on the sum of the neutrino masses $\Sigma m_{\nu}$ of

$$
\frac{\sigma\left(\Sigma m_{\nu}\right)}{\mathrm{meV}}= \begin{cases}103 & (\mathrm{P} 15+\mathrm{BAO}-15) \\ 44 & (\mathrm{~S} 3 \text {-wide }+\mathrm{BAO}-15) \\ 22 & (\mathrm{~S} 3 \text {-wide }+\mathrm{DESI}) \\ 19 & {[\mathrm{~S} 4(\ell>50)+\mathrm{DESI}]} \\ 15 & {[\mathrm{~S} 4(\ell>5)+\mathrm{DESI}]}\end{cases}
$$

where S3 and S4 include Planck-pol at large scales. If we replace S3-wide with the S3-deep survey we find $\sigma\left(\Sigma m_{\nu}\right)=$ $53 \mathrm{meV}$ combined with BAO-15, and $25 \mathrm{meV}$ with DESI. The expected constraints are summarized in Fig. 3, and are consistent with findings in $\mathrm{Wu}$ et al. [31]. These forecasts imply that if the neutrino hierarchy is inverted, with mass sum $>100 \mathrm{meV}$, we may have $>2 \sigma$ evidence for nonzero mass in the next few years from S3 data, and an almost $5 \sigma$ detection in $\approx 5$ years with DESI. If instead it is the minimal mass of $60 \mathrm{meV}$, a $2 \sigma-3 \sigma$ indirect measurement should be reachable in five years, with stronger evidence from the subsequent experiments.

As illustrated in Fig. 4, there is a strong positive correlation between the neutrino mass and cold-dark-matter density in the CMB observables. This arises predominantly from the competing influence of these parameters on the lensing signal. Increased neutrino mass suppresses smallscale power, while increasing cold-dark-matter density boosts small-scale power by shifting matter-radiation equality to earlier times; this shortens the radiationdomination epoch in which subhorizon modes of the gravitational potential decay, enhancing the small-scale amplitude of structure $[29,60,61]$. Conversely, BAO data
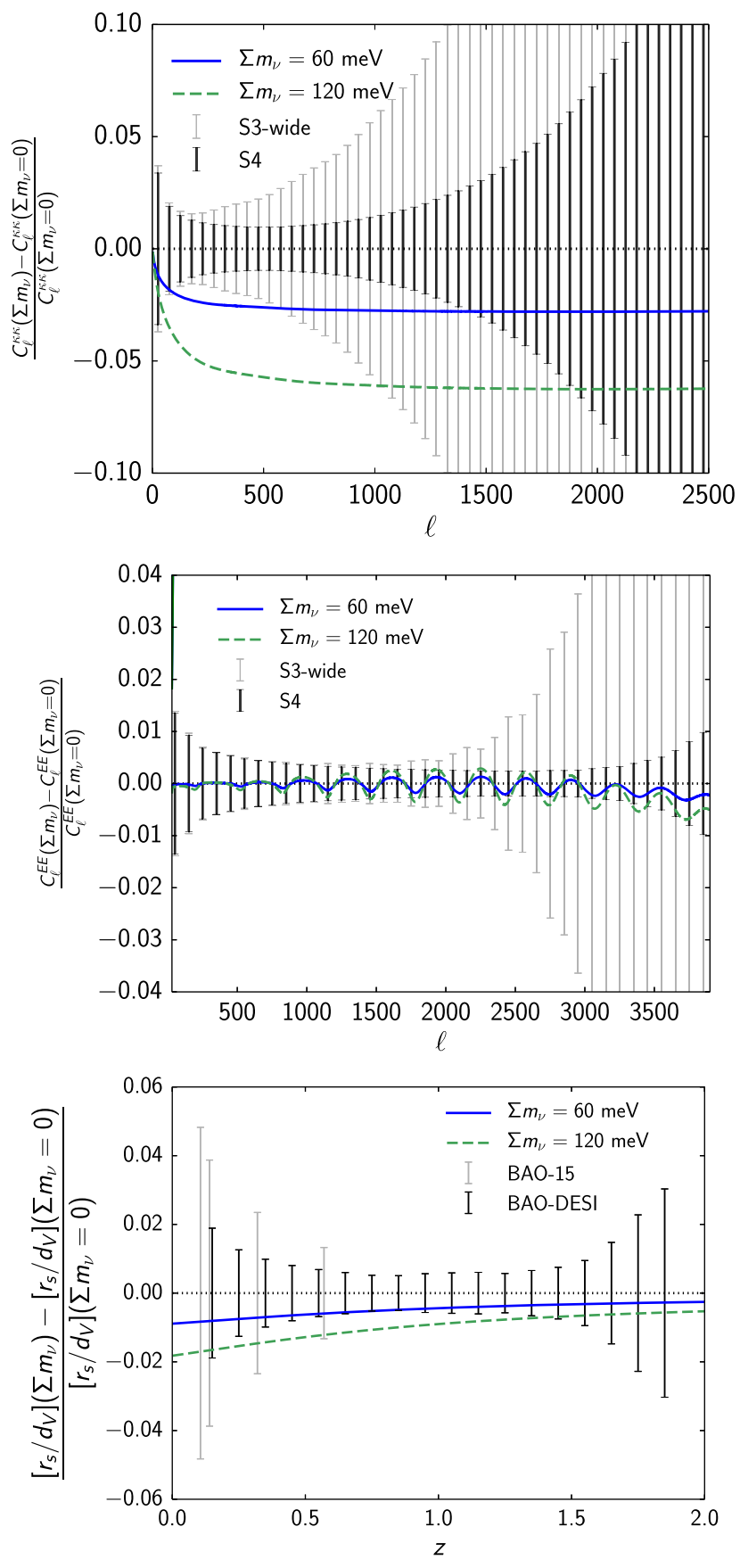

FIG. 2 (color online). Top and middle: Fractional change in the convergence $\kappa$ (top) and $E$-mode (middle) power spectrum with neutrino mass, for fixed $\Omega_{\mathrm{c}} h^{2}+\Omega_{\nu} h^{2}$, with expected uncertainties for S3-wide and S4 CMB data. A higher neutrino mass has less lensing, decreasing the $E$-mode peak smoothing. Bottom: Fractional change in distance ratio $r_{s} / d_{V}$, with uncertainties from current (BAO-15 [20]) and forecast (DESI [25]) BAO data. Here $\Omega_{\mathrm{c}} h^{2}$ is fixed.

constrain the sum of the CDM and massive neutrino density, since it is this combination that affects the angular diameter distance and expansion rate.

The power of the BAO and CMB data lie in their combination. BAO measurements alone cannot constrain 


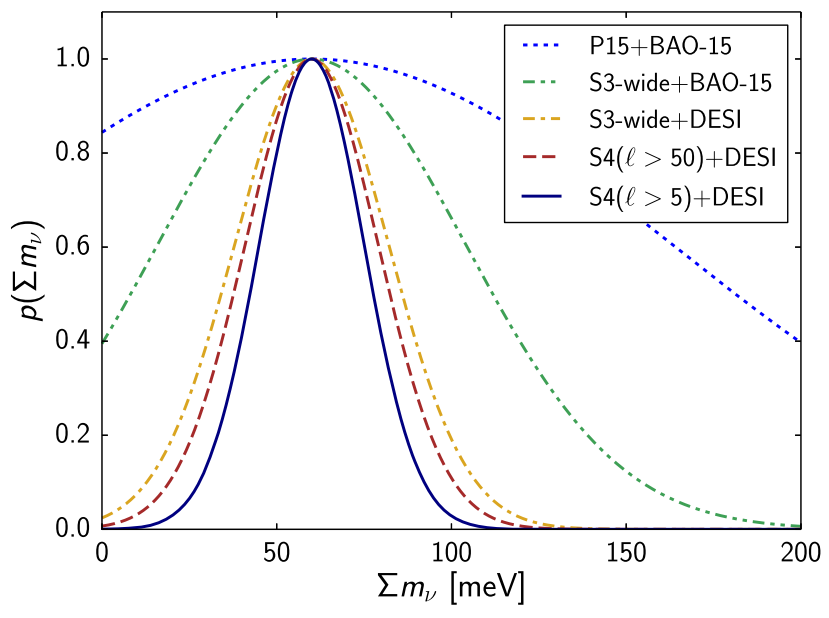

FIG. 3 (color online). Forecast marginal posterior constraints on the sum of the neutrino masses $\Sigma m_{\nu}$ within a $\Lambda \mathrm{CDM}+\Sigma m_{\nu}$ model, assuming Gaussian error distributions. The current uncertainties (P15 + BAO-15) are expected to improve rapidly, with S3 CMB data and DESI BAO data expected by $\sim 2020$.

the primordial parameters $A_{s}$ and $n_{s}$, nor the optical depth to reionization $\tau$; the constraints lie in the four-dimensional subspace spanned by $\Omega_{c} h^{2}, \Omega_{b} h^{2}, \Sigma m_{\nu}$, and $\theta_{A}$ (being most constraining in the directions corresponding approximately to $\Omega_{m} h^{2}$ and $\theta_{A}$ ). S4 alone could achieve a neutrino mass error of $\sigma\left(\Sigma m_{\nu}\right)=53 \mathrm{meV}$; with DESI BAO this is expected to tighten to $\sigma\left(\Sigma m_{\nu}\right)=19 \mathrm{meV}$, enough for a $3 \sigma$ detection of the minimal mass.

For comparison, we note that the forecast conditional neutrino mass uncertainty (the uncertainty when fixing the $\Lambda \mathrm{CDM}$ parameters) is $\sigma\left(\Sigma m_{\nu}\right)=3 \mathrm{meV}$ for $\mathrm{S} 4(\ell>5)+\mathrm{DESI}$, compared to $\sigma\left(\Sigma m_{\nu}\right)=15 \mathrm{meV}$ in

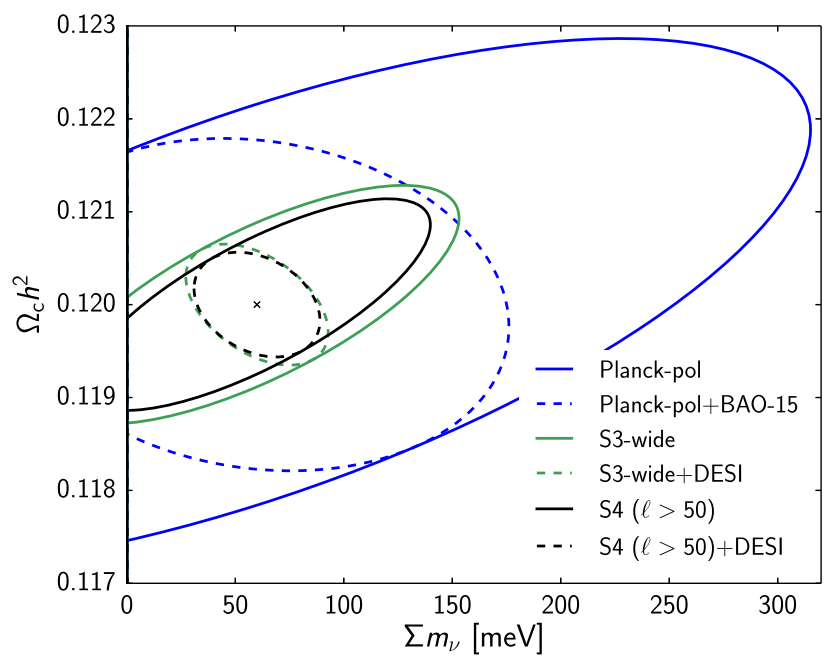

FIG. 4 (color online). Expected joint constraint (68\% C.L.) on the neutrino mass sum $\Sigma m_{\nu}$ and physical cold-dark-matter density $\Omega_{\mathrm{c}} h^{2}$ within a $\Lambda \mathrm{CDM}+\Sigma m_{\nu}$ model. The BAO constraint, sensitive to the total late-time cold-dark-matter density, is almost orthogonal to the CMB lensing constraint, breaking the degeneracy. the marginalized case. This highlights the effect of degeneracies even in the base model.

Comparing to previous results, the $\mathrm{S} 4(\ell>5)+$ DESI forecast matches previous results [30-32], and the "current data" P15 + BAO-15 forecast is compatible with the published result: $\sigma\left(\Sigma m_{\nu}\right)<0.23 \mathrm{eV}$ at $95 \%$ confidence [21], with the forecast errors on other $\Lambda \mathrm{CDM}$ parameters also matching closely.

Here we choose by default a fiducial mass sum of $60 \mathrm{meV}$, approximating the normal hierarchy as one massive neutrino and two massless neutrinos, with a modified temperature to account for QED and noninstantaneous decoupling effects (giving $N_{\text {eff }}=3.046$ ). Given the known mass splittings from oscillation experiments, this is an excellent approximation to the normal hierarchy in the minimal-mass scenario [4]. For comparison, we compute the constraint on the neutrino mass sum in the inverted hierarchy, with two degenerate massive neutrinos and one massless neutrino, with a neutrino mass sum of $\Sigma m_{\nu}=120 \mathrm{meV}$. For these two cases we find $\sigma\left(\Sigma m_{\nu}\right)=53(54) \mathrm{meV}$ for $\mathrm{S} 4$ alone for a fiducial mass of 60 (120) meV; with DESI included this reduces to $\sigma\left(\Sigma m_{\nu}\right)=19(20) \mathrm{meV}$. We therefore do not expect our results are sensitive to the adopted fiducial model. We describe further tests of the code in the Appendix.

We consider the impact of using an improved, likelihood-based lensing estimator (going beyond the firstorder quadratic estimator of $\mathrm{Hu}$ and Okamoto [58]) to reduce the effective noise power $N_{l}^{\kappa \kappa}$ of the lensing reconstruction. Hirata and Seljak [62] show that $N_{l}^{\kappa \kappa}$ can be reduced by a factor of 2 for a S4-like experiment; under this modification, we find only a $3 \%$ tightening of the neutrino mass constraint for S4 + DESI. The improvement is small due to the contribution of cosmic variance $(\mathrm{CV})$ to the lensing power spectrum uncertainties, and the degeneracy of neutrino mass with other $\Lambda C D M$ parameters; the S4 lensing power spectrum derived from the quadratic estimator is already CV-limited out to $\ell \approx 800$.

As in Benoit-Lévy et al. [40], we find that there is useful information in the lensed power spectra. We show the relative impact on forecasted constraints for $\mathrm{S} 3$-wide and S4 in Table II (including Planck-pol at large scales but no BAO). Lensing reduces the neutrino mass uncertainty by a factor of $\approx 6$ compared to the unlensed CMB. As such, the two-point functions will provide increasingly more information as $E$-mode polarization measurements

TABLE II. Impact of lensing on the neutrino mass constraint (in units of meV). Constraining power comes from both the lensed spectra (two-point) and the reconstructed lensing potential (fourpoint). Gaussian uncorrelated errors are assumed.

\begin{tabular}{|c|c|c|c|}
\hline & Unlensed & $\begin{array}{l}\text { Lensed } T T, T E, E E \\
\quad \text { (two-point only) }\end{array}$ & $\begin{array}{c}\text { Unlensed }+\kappa \kappa \\
\text { (four-point only) }\end{array}$ \\
\hline $\mathrm{S} 3, \sigma\left(\Sigma m_{\nu}\right)$ & 435 & 75 & 61 \\
\hline $\mathrm{S} 4, \sigma\left(\sum m_{\nu}\right)$ & 363 & 64 & 53 \\
\hline
\end{tabular}


improve. Checking for consistency between the lensed observables will be an important systematic test for new CMB data. For example, marginalizing over the lensing effect in the two-point functions, by introducing a variable lensing amplitude parameter $A_{\text {lens }}$ [63], would isolate the impact of neutrinos on the four-point function.

\section{DEPENDENCE ON EXPERIMENTAL DETAILS}

Since future CMB experiments are currently under development, we investigate the importance of certain experimental details on the mass constraint.

\section{A. Importance of the reionization bump}

The amplitude of primordial power, $A_{s}$, is partially degenerate with $\Sigma m_{\nu}$, since $A_{s}$ increases the amplitude of clustering at small scales, and $\Sigma m_{\nu}$ decreases it in an approximately scale-independent fashion (e.g., Fig. 2). The amplitude $A_{s}$ is not well determined by the primordial CMB temperature anisotropy; an increased optical depth to reionization lowers the signal such that the normalization of the anisotropy measures the combination $A_{s} e^{-2 \tau}$ [64]. This leads to a degeneracy between $\tau$ and $\Sigma m_{\nu}$ that can be broken with precision measurements of the reionization bump at multipoles $\ell<20$ in polarization [25,65].

Here we explore the importance of making a robust optical depth measurement, considering three cases for S4: current WMAP measurements $[42,66]$, optimistic future Planck-pol measurements (see Appendix), and a future S4 measurement that reaches the largest scales $\left(\ell_{\min }=5\right)$. We find forecast constraints of

$\frac{\sigma\left(\Sigma m_{\nu}\right)}{\mathrm{meV}}= \begin{cases}27 & {[\mathrm{~S} 4(\ell>50)+\text { WMAP-pol }+\mathrm{DESI}]} \\ 19 & {[\mathrm{~S} 4(\ell>50)+\text { Planck-pol }+\mathrm{DESI}]} \\ 15 & {[\mathrm{~S} 4(\ell>5)+\mathrm{DESI}]}\end{cases}$

with the uncertainty on $\tau$ reducing from 0.008 to 0.005 to 0.003 , respectively. This is compared to 0.013 for WMAPpol from $E E$ alone; i.e., improved CMB lensing data helps constrain $\tau$ even when the neutrino mass is varied. Figure 5 shows the expected correlation between $\tau$ and neutrino mass.

Figure 5 also shows the impact of reducing the minimum multipole of the S4 experiment on the neutrino mass constraint, supplemented with Planck-pol or the current WMAP-pol at the largest scales. There is a limiting plateau for $\mathrm{S} 4$ at $\ell_{\min }>20$, and a clear improvement as the polarization is better measured at increasingly large scales. The $\mathrm{S} 4(\ell>5)+$ DESI limit reaches the $\mathrm{CV}$ limit for CMB data. ${ }^{1}$

\footnotetext{
${ }^{1}$ Pan and Knox [32] found that a S4 experiment combined with a CV-limited BAO experiment could tighten the neutrino mass constraint further, to $\sigma\left(\Sigma m_{\nu}\right)=11 \mathrm{meV}$.
}
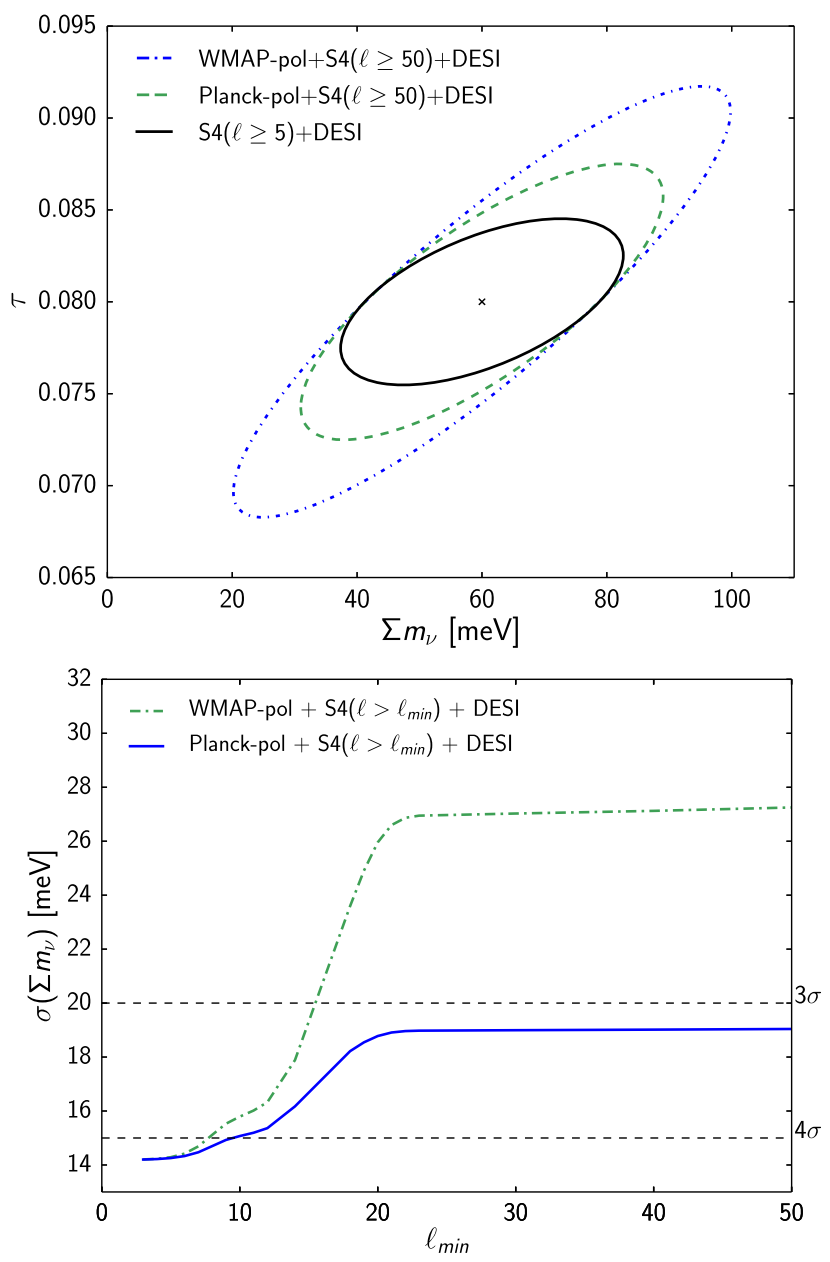

FIG. 5 (color online). Top: The neutrino mass $\Sigma m_{\nu}$ is correlated with the optical depth to reionization $\tau$ (forecast 68\% C.L.). Current data at $\ell<20$ (WMAP-pol) would leave a degeneracy between $\Sigma m_{\nu}$ and $\tau$ that could be broken with improved largescale polarization data. Bottom: The expected neutrino mass constraint as a function of the minimum multipole accessible to $\mathrm{S} 4$, indicating the benefit of reaching large scales.

We then also consider the relative importance of making a higher sensitivity small-scale measurement, versus a new large-scale polarization measurement. We start with a S3-type $\ell>50$ experiment, and then either increase the $\ell>50$ sensitivity, or supplement it with a new CV-low large-scale measurement at $\ell<50$. We find forecast constraints of

$$
\frac{\sigma\left(\Sigma m_{\nu}\right)}{\mathrm{meV}}= \begin{cases}22 & {[\mathrm{~S} 3(\ell>50)+\text { Planck-pol }+\mathrm{DESI}]} \\ 19 & {[\mathrm{~S} 4(\ell>50)+\text { Planck-pol }+\mathrm{DESI}]} \\ 17 & {[\mathrm{~S} 3(\ell>50)+\text { CV-low }+\mathrm{DESI}]}\end{cases}
$$

This indicates that a cosmic-variance-limited measurement of optical depth could be more valuable than more sensitive small-scale data, especially given that Planck-pol large-scale 
polarization data is itself not yet demonstrated to be free of systematic errors.

We note that $21-\mathrm{cm}$ experiments, which map the brightness temperature of neutral hydrogen as a function of redshift, will probe the epoch of reionization [67]; the combination of this information with $\mathrm{CMB}+\mathrm{BAO}$ would break the $\Sigma m_{\nu}-\tau$ degeneracy and improve the neutrino mass constraint (investigated in Liu et al. [68]).

\section{B. Importance of sensitivity and angular range}

For the particular case of an $\ell>50$ experiment covering $40 \%$ of the sky at 3 -arcmin resolution, combined with Planck-pol, we vary the white-noise sensitivity. The forecast neutrino mass limits are shown in Fig. 6 for CMB-only, $\mathrm{CMB}+\mathrm{BAO}-15$, and $\mathrm{CMB}+\mathrm{BAO}-\mathrm{DESI}$. There is clearly an improvement as the noise is reduced, and a significant gain is expected over current Planck measurements, but below white $=$ noise levels of $\approx 10 \mu \mathrm{K}$-arcmin there does not appear to be a substantial gain (as also seen in $\mathrm{Wu}$ et al. [31]).

It is not yet certain whether this $\approx 10 \mu \mathrm{K}$-arcmin noise level, over half the sky, will be achieved in practice from the upcoming S3 CMB experiments, or whether the lensing reconstruction will achieve the expected noise levels. Atmospheric, ground, and foreground emission are typical contaminants that would increase the effective noise in the maps and in the lensing reconstruction. New data from the current S2 experiments will help clarify the impact of nonwhite noise on the lensing noise performance.

Here we have continued to restrict our analysis to "clean" scales at $\ell<3000$ in $T T$ and $T E$ and $\ell<4000$ in EE. A foreground-free $\mathrm{S} 3$ or $\mathrm{S} 4$ experiment would contain information from $\ell>3000$; the TT spectrum is signal dominated to small scales $(\ell \approx 4800$ for S4) where there are a large number of modes. However, uncertainty about extragalactic foregrounds will likely make this information

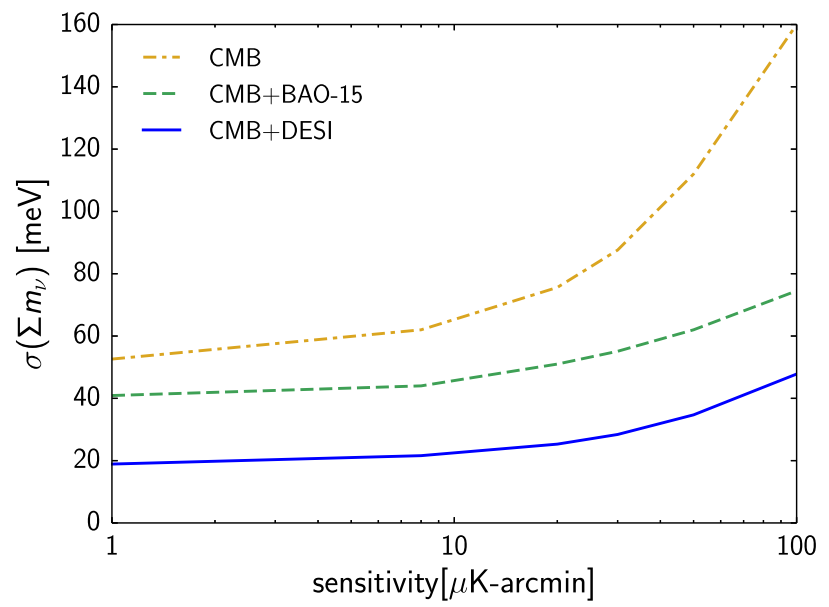

FIG. 6 (color online). The dependence of the neutrino mass constraint $\sigma\left(\Sigma m_{\nu}\right)$ on CMB map sensitivity, for a 3-arcmin resolution experiment covering $40 \%$ of the sky. inaccessible. We find that including $\ell>3000$ scales would tighten the neutrino mass error by $\approx 10 \%$.

We restrict the modes available for reconstruction of the lensing potential to these same scales, and to $\ell>50$, since reconstruction on the largest scales has yet to be demonstrated (there are difficulties such as mean-field subtraction for masked fields [69]). We find that the information, quantified by the term $F_{\ell}^{\Sigma m_{\nu}}$ in the Fisher matrix, is concentrated in the multipole range $100 \lesssim \ell \lesssim 1000$ for S3 and $200 \lesssim \ell \lesssim 2000$ for S4. This also means that detailed nonlinear modeling should not be required, since at redshift $z \approx 2$ at the peak of the CMB lensing kernel [Eq. (4)], the information peak corresponds to physical scales $\gtrsim 200 \mathrm{Mpc}$.

\section{HOW UNIQUE IS THE MASSIVE NEUTRINO SIGNAL?}

The measurable effect of neutrinos can be partly mimicked by changes in other cosmological parameters, as explored in, e.g., Font-Ribera et al. [25], Benoit-Lévy et al. [40], Hamann et al. [70]. Massive neutrinos affect the expansion rate and angular diameter distance, but changes in curvature, dark-energy history or the Hubble parameter can compensate to keep the well-constrained acoustic peak positions and structure essentially unchanged.

Here we consider changes in the spatial curvature and changes in the energy density of dark energy with time. Dark energy is invoked to explain the acceleration of the Universe, and may take the form of a cosmological constant with constant energy per unit proper volume, although an evolving dark-energy equation of state $(w \neq-1)$ is not ruled out by current observations [21]. We consider two parameterizations for dark energy; these are the usual Taylor expansion in the scale factor for dynamical dark energy [71,72],

$$
w(a)=w_{0}+w_{a}(1-a),
$$

with two free parameters $w_{0}$ and $w_{a}$, and also the Doran and Robbers [73] model for early dark energy,

$$
\Omega_{\Lambda}(a)=\frac{\Omega_{\Lambda}-\Omega_{e}\left(1-a^{-3 w_{0}}\right)}{\Omega_{\Lambda}+\Omega_{m} a^{3 w_{0}}}+\Omega_{e}\left(1-a^{-3 w_{0}}\right),
$$

with parameters $\Omega_{e}$ and $w_{0}$. This has a background expansion similar to a massive neutrino for periods of the evolution of the universe. Previous work has considered neutrino mass constraints within this model (e.g., [74,75]). We use the CAMB Parameterized Post-Friedmann (PPF) module [76] and a modified version of CAMB from Calabrese et al. [74] to compute the power spectrum within these models.

We take as our baseline the $S 4(\ell>50)+$ DESI experiment. Marginalizing over simple extensions to the $\Lambda \mathrm{CDM}+\Sigma m_{\nu}$ model, we find 


$$
\frac{\sigma\left(\Sigma m_{\nu}\right)}{\mathrm{meV}}= \begin{cases}19 & \left(\Lambda \mathrm{CDM}+\Sigma m_{\nu}\right) \\ 30 & \left(\Lambda \mathrm{CDM}+\Sigma m_{\nu}+\Omega_{k}\right) \\ 27 & \left(\Lambda \mathrm{CDM}+\Sigma m_{\nu}+w_{0}\right) \\ 46 & \left(\Lambda \mathrm{CDM}+\Sigma m_{\nu}+w_{0}+w_{a}\right) \\ 37 & \left(\Lambda \mathrm{CDM}+\Sigma m_{\nu}+\Omega_{e}+w_{0}\right) \\ 64 & \left(\Lambda \mathrm{CDM}+\Sigma m_{\nu}+w_{0}+w_{a}+\Omega_{k}\right) .\end{cases}
$$

We discuss these parameter degeneracies in the remainder of this section. We find that marginalizing over the neutrino number $N_{\text {eff }}$ (and other extension parameters that modify the primordial $\mathrm{CMB}$ spectrum such as a running spectral index) have a $<10 \%$ effect on the predicted neutrino mass uncertainties. These findings are summarized in Fig. 7, which also includes the corresponding S3 forecasts.

At first sight, the degradation of the neutrino mass estimate in the case of varying $w_{0}, w_{a}$, and $\Omega_{k}$ simultaneously appears severe, more than tripling the error bar. However, this model has three extra parameters compared to $\Lambda \mathrm{CDM}$; within the Bayesian framework, one can rigorously ask whether additional parameters are required by the data, quantifying the trade-off between improving the fit against an increased complexity of the model (see, e.g., [77] for discussion in the context of cosmological data). This model selection approach would quantify the need for additional extension parameters, and would disfavor an overparameterized model if it is not required by the data. In practice our challenge is likely to lie in distinguishing between different one-parameter extensions to $\Lambda \mathrm{CDM}$ : Are we seeing nonzero neutrino mass, or could

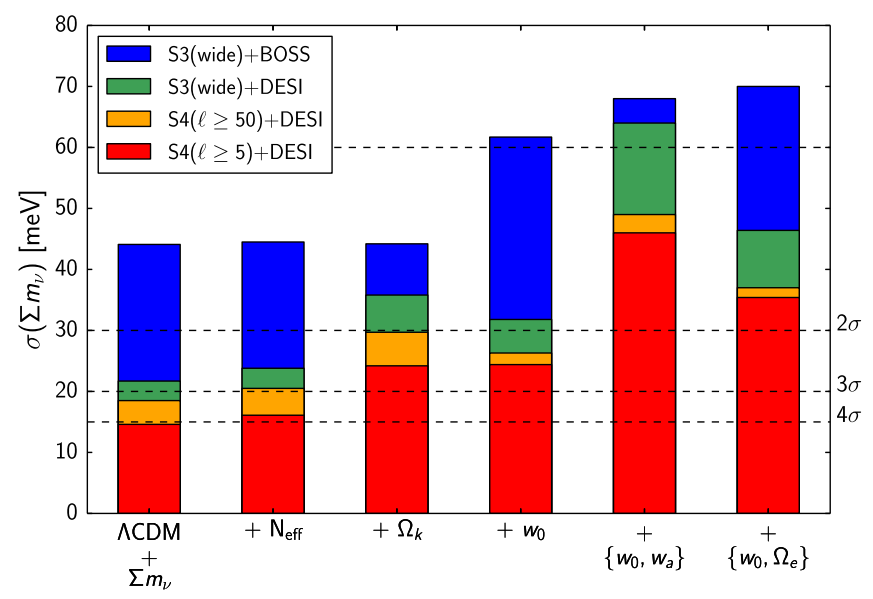

FIG. 7 (color online). Neutrino mass constraints forecast for different data combinations and simple one- or two-parameter extensions to the $\Lambda \mathrm{CDM}+\Sigma m_{\nu}$ model [all except $\mathrm{S} 4(\ell \geq 5)$ include Planck-pol information at low $\ell$ ]. With $\mathrm{CMB}+\mathrm{BAO}$ data, neutrino mass is not correlated with the number of neutrino species, $N_{\text {eff }}$, but is partly correlated with spatial curvature, $\Omega_{k}$, and with the dark energy equation of state, $w_{0}$. Expected confidence levels are shown assuming the minimal total neutrino mass $\Sigma m_{\nu}=60 \mathrm{meV}$. it be similarly well explained by a small amount of curvature, or a small deviation from a $w=-1$ equation of state?

\section{A. Physical degeneracies}

To help understand these degeneracies, we note the Hubble parameter $H(z)$ is given by

$$
\begin{aligned}
{\left[\frac{H(z)}{H_{0}}\right]^{2}=} & \Omega_{r}(1+z)^{4}+\Omega_{m}(1+z)^{3} \\
& +\Omega_{k}(1+z)^{2}+\Omega_{\Lambda}(z),
\end{aligned}
$$

at times after the neutrinos become nonrelativistic, where $\Omega_{\Lambda}(z)$ is the dark-energy density, constant for $w=-1, \Omega_{r}$ is the radiation density today (e.g., photons and massless neutrinos), $\Omega_{m}=\Omega_{c}+\Omega_{b}+\Omega_{\nu}$ is the matter density today (CDM, baryons, and massive neutrinos) and $\Omega_{\nu} h^{2}=$ $\Sigma m_{\nu} / 93 \mathrm{eV}$ is the physical massive neutrino density today. The angular diameter distance is given by

$$
d_{A}(z)=\frac{c}{H_{0}(1+z)} \begin{cases}\frac{1}{\sqrt{-\Omega_{k}}} \sin \left(\sqrt{-\Omega_{k}} \int_{0}^{z} \frac{H_{0} d z^{\prime}}{H\left(z^{\prime}\right)}\right) & \Omega_{k}<0, \\ \int_{0}^{z} \frac{H_{0} d z^{\prime}}{H\left(z^{\prime}\right)} & \Omega_{k}=0, \\ \frac{1}{\sqrt{\Omega_{k}}} \sinh \left(\sqrt{\Omega_{k}} \int_{0}^{z} \frac{H_{0} d z^{\prime}}{H\left(z^{\prime}\right)}\right) & \Omega_{k}>0 .\end{cases}
$$

Considering curvature, photons propagating in a nonflat universe follow curved geodesics, changing the angular diameter distance to an object of fixed proper size, at a given comoving distance, relative to a flat universe. Varying curvature shifts the angular scale of the acoustic peaks; to remain consistent with the CMB data, the matter density and Hubble constant $H_{0}$ must vary to keep the peak structure unchanged. This is the well-known geometric degeneracy [78].

This degeneracy is partially broken by CMB lensing measurements [79], which are sensitive to the growth of structure in the late-time universe and, therefore, to the matter density $\Omega_{m}$ and dark-energy density $\Omega_{\Lambda}$. For a fixed CMB acoustic peak scale, the effect of decreasing the curvature parameter $\Omega_{k}$, moving to a more closed universe, is to decrease the Hubble constant and increase $\Omega_{m}$. This enhances the amplitude of the CMB lensing power spectrum, as illustrated in Fig. 8, which can be compensated by increasing $\Sigma m_{\nu}$. This leads to an anticorrelation between $\Sigma m_{\nu}$ and $\Omega_{k}$ when using CMB measurements alone, as shown in Fig. 9.

The BAO constraint in the $\Sigma m_{\nu}-\Omega_{k}$ plane is approximately orthogonal to the CMB-only constraint, because decreasing $\Omega_{k}$ decreases the volume distance to a given redshift [Eq. (5)]. This can be compensated by a smaller matter density, lowering the neutrino mass. These data are 


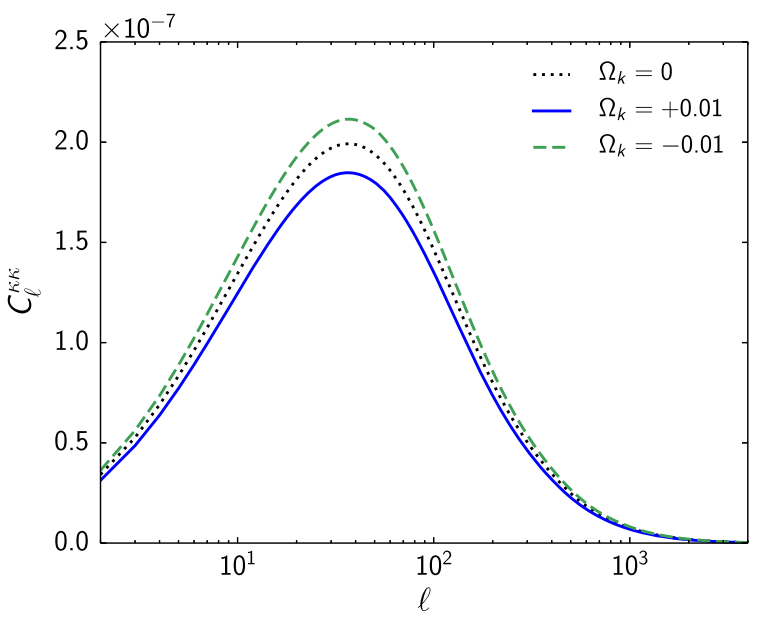

FIG. 8 (color online). CMB convergence power spectrum for varying $\Omega_{k}$, with other parameters holding the primary CMB fixed. Decreasing $\Omega_{k}$ requires a smaller Hubble constant $H_{0}$ and increased growth rate. This has a similar effect to decreasing the neutrino mass.

powerful in combination, and for S3 + BOSS the neutrino mass constraint is independent of curvature. However, the neutrino mass constraint from S3 + DESI or S4 + DESI is expected to degrade by $\approx 50 \%$ when allowing for curvature, as illustrated in Fig. 7.

Similar arguments apply to dark energy, which modifies the background evolution according to its equation of state, but does not contribute to clustering. Increasing the darkenergy equation-of-state parameter $w_{0}$ leads to an increased expansion rate, shifting the angular scale of the acoustic peaks; to remain consistent with the CMB data, the Hubble constant $H_{0}$ decreases to keep the peak structure unchanged. Similar to the curved model, this increases the clustering, which can be compensated with larger neutrino masses. This gives the positive correlation between $w_{0}$ and $\Sigma m_{\nu}$ in CMB data, illustrated in Fig. 9 and reported in, e.g., $[40,80]$. The BAO degeneracy is, however, also positively correlated, so the neutrino mass uncertainty is inflated more than when allowing for curvature. Increasing $\Sigma m_{\nu}$ increases the contribution of neutrinos to $\Omega_{m}$, requiring a smaller $\Omega_{\Lambda}$ (in a flat universe); the volume distance to a given redshift, and, hence, the BAO peak position, can then be preserved by increasing $w_{0}$. The early dark-energy density parameter, $\Omega_{e}$, is anticorrelated with neutrino mass, due to their similar effects on the background expansion $[74,75] .^{2}$

We find that the neutrino mass constraints from S3 + DESI or S4 + DESI are degraded by more than a factor of 2 when allowing for a nonminimal $(w \neq-1)$

\footnotetext{
${ }^{2}$ Here we adopted a fiducial $\Omega_{e}=0.007$, allowed by current data [81]. A smaller $\Omega_{e}$ would improve the neutrino mass constraint, as the parameters are anticorrelated and $\Omega_{e}$ cannot be negative.
}
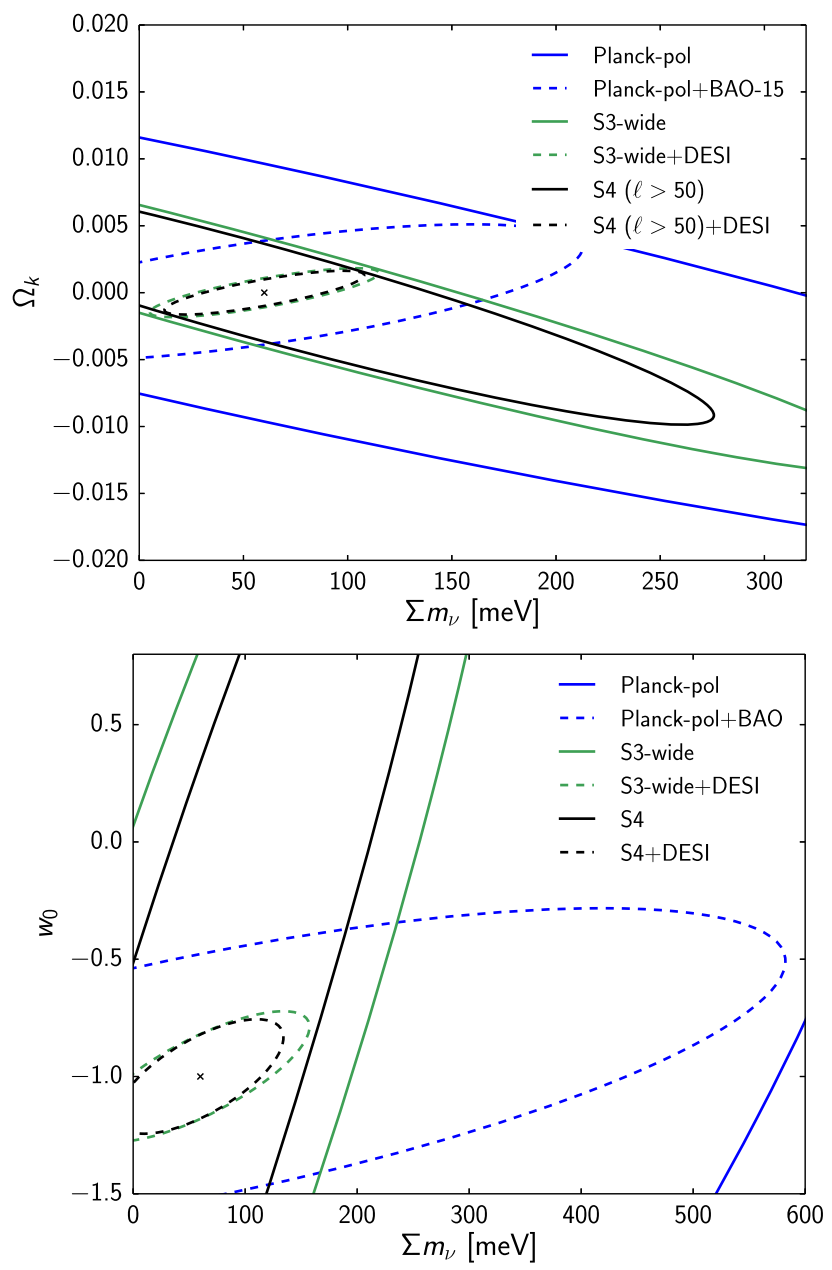

FIG. 9 (color online). Top: Forecast joint constraint on the neutrino mass, $\Sigma m_{\nu}$, and spatial curvature, $\Omega_{k}$, within a $\Lambda \mathrm{CDM}+$ $\Sigma m_{\nu}+\Omega_{k}$ model. The BAO data breaks the anticorrelated degeneracy in the CMB data. Bottom: Forecast constraint on $\Sigma m_{\nu}$ and the dark energy equation of state, $w_{0}$, marginalized over the $\Lambda \mathrm{CDM}$ and $w_{a}$ parameters.

dark-energy equation of state in the cosmological model, as shown in Fig. 7. Distinguishing a nonzero neutrino mass parameter from a universe with dark energy beyond the cosmological constant will be difficult with the $\mathrm{CMB}+\mathrm{BAO}$ data combinations considered here. Allowing for freedom in both dark energy and curvature (i.e., a $\Lambda \mathrm{CDM}+\Sigma m_{\nu}+\Omega_{k}+w_{0}+w_{a}$ model) degrades the constraint further to $\sigma\left(\sum m_{\nu}\right)=64 \mathrm{meV}$ for S4 + DESI, but would include three new parameters.

\section{B. Breaking degeneracies with complementary measurements}

We have focused so far on the minimal combination of future $\mathrm{CMB}$ and $\mathrm{BAO}$ data. To break these remaining degeneracies between neutrino mass and curvature/darkenergy parameters, we would turn to other large-scale structure probes such as the galaxy power spectrum, 


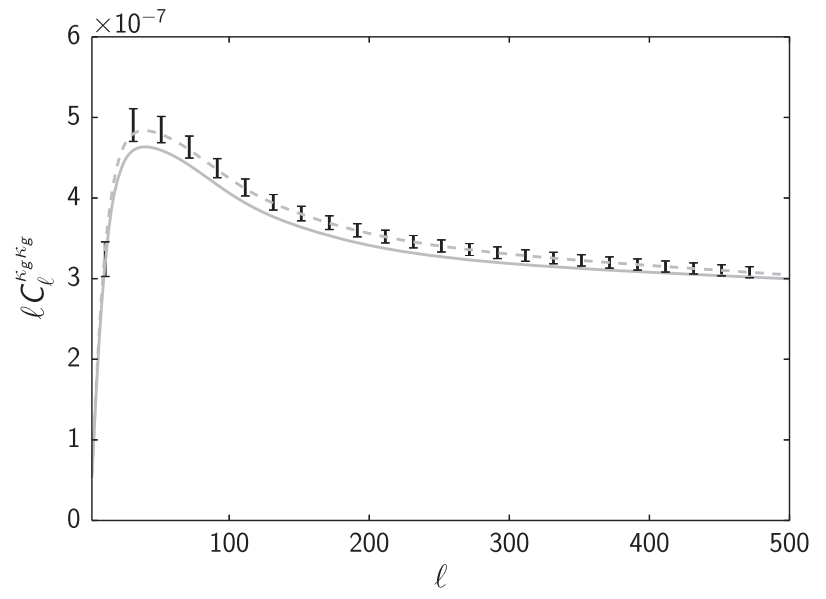

FIG. 10. Galaxy lensing power spectra for two models degenerate in CMB spectra and BAO distance ratios. They could be distinguished using a future galaxy weak lensing survey $\left[f_{\text {sky }}=0.5, n_{\text {gal }}=10 \operatorname{arcmin}^{-2}\right.$, and source redshift distribution $n(z)$ as for the LSST "gold sample" [83] ].

redshift-space distortions, galaxy weak lensing, the kinematic Sunyaev-Zel'dovich effect, and galaxy cluster counts, which measure the growth of structure at later times, and Type Ia supernovae, which better constrain the expansion rate.

We have not included these and other data in our baseline forecasts as they are, arguably, more prone to systematic uncertainties such as tracer bias and the shape of the nonlinear power spectrum, which contains unknown baryonic feedback effects, source distribution uncertainties, and multiplicative bias $[21,37,82]$. However, a promising path would be to examine a suite of different complementary probes, each in combination with the CMB and $\mathrm{BAO}$ data, to distinguish neutrino mass from a nonflat or non- $\Lambda$ model.

For example, in Fig. 10 we illustrate schematically how galaxy weak lensing can break remaining degeneracies between two example models. Here the CMB spectra and BAO distances are indistinguishable with $\mathrm{S} 4+$ DESI data (see parameters in the Appendix).

Their galaxy weak lensing signals, which probe the growth of structure during the dark-energy-dominated era, are distinct, and should be distinguishable with a plausible future weak lensing survey. With a $2 \%$ difference in $\sigma_{8}$, robust galaxy cluster measurements should also help discriminate between these models (e.g., $[84,85])$.

\section{DISCUSSION AND CONCLUSIONS}

We have demonstrated that an indirect detection of the sum of the neutrino masses should be possible with upcoming $\mathrm{CMB}$ and $\mathrm{BAO}$ surveys. In the next decade a $4 \sigma$ detection should be reachable, within the $\Lambda \mathrm{CDM}+\Sigma m_{\nu}$ model, even in the minimal mass $\left(\Sigma m_{\nu}=60 \mathrm{meV}\right)$ scenario. We have found that this is contingent on obtaining improved large-scale polarization measurements from the $\mathrm{CMB}$, which may be the hardest experimental challenge. It will also be necessary to exclude degeneracies with other plausible extensions such as curvature and dark energy. We find that allowing for these extensions degrades the expected neutrino mass constraint, and that use of other large-scale structure probes will be necessary to rule out other departures from $\Lambda \mathrm{CDM}$.

Our forecasts make a number of assumptions. We have neglected non-Gaussian terms in the CMB covariance due to correlation of the temperature, polarization, and lensing fields, which should have a small impact on imminent data but will become more important as noise levels reduce. We assume Gaussianity of the posterior distribution and rely on data independence of the covariance matrix. These assumptions become increasingly accurate for the precision future experiments considered here, but remain an approximation. Our analysis also assumes little foreground contamination. This will be most important for the large-scale CMB signal, but is not expected to significantly degrade the smallerscale lensing signal.

We have assumed white noise in the new CMB data. This has not yet typically been achieved in practice from the ground, due to atmospheric and scan-synchronous emission that induces additional variance at large scales. However, polarization measurements are cleaner than temperature in this respect, and new experiments have sophisticated designs to modulate the polarization signal. Performance of current Stage-2 experiments will help refine noise projections for the future experiments. We have also assumed ideal performance of the future DESI $\mathrm{BAO}$ experiment.

In terms of theoretical scope, beyond an evolving darkenergy equation of state, we have not considered other possible new physics. For instance, theoretically motivated axion contributions to the background expansions and perturbations might offer an alternative explanation for a neutrino mass detection. The cosmological effects are not identical [86], but further investigation will be useful.

Finally, neutrino mass is the next beyond- $\Lambda \mathrm{CDM}$ parameter that we know will be needed to fit data, so it is valuable that competitive constraints are expected to come from different combinations of cosmological data sets beyond the CMB/BAO considered here. Their complementarity will aid in convincingly excluding systematic effects and alternative cosmological models.

\section{ACKNOWLEDGMENTS}

We thank David Alonso, Anthony Challinor, Pedro Ferreira, and Blake Sherwin for useful discussions. Some similar forecasts were done for the Advanced ACT proposal; we thank the ACT team for valuable input. R. A., J.D. and T.L. are supported by ERC Grant No. 259505, and E. C. is supported by STFC. P.C. is grateful to the University of Oxford for its hospitality while 
part of this work was done, and to the École Normale Supérieure for financial support.

\section{APPENDIX: FORECASTING PARAMETER CONSTRAINTS}

We forecast posterior parameter constraints and degeneracy directions by evaluating the relevant Fisher matrix as defined in Eq. (6). We assume uniform priors on all parameters, allowing the posterior $p(\boldsymbol{\theta})$ to be replaced with the likelihood $\mathcal{L}(\boldsymbol{\theta})=\mathbb{P}(\mathbf{d} \mid \boldsymbol{\theta}, M)$ in Eq. (6). The Fisher matrix is evaluated at fiducial parameters; the choice is unimportant under the assumption of a Gaussian posterior and data-independent covariance, although in practice they are chosen to match the current best-fit parameters of the model, as the real covariance does have a term that scales with the signal. Our choices are shown in Table III.

To compute the Fisher matrix we use as observables the $\mathrm{CMB}$ power spectra and the $\mathrm{BAO}$ distance measurements. For the CMB, we use the lensed power spectrum between each pair of (assumed Gaussian) fields $X, Y$ from their spherical harmonic coefficients,

TABLE III. Fiducial values and step sizes for the numerical derivatives, chosen to be small enough to minimize error in the Taylor expansion of the two-sided derivative, while keeping numerical stability in the derivatives from the CAMB power spectra. The fiducial value of $\Omega_{e}=0.007$ is at the upper $95 \%$ confidence level given current data [81].

\begin{tabular}{lcc}
\hline \hline Parameter & Fiducial value & Step size \\
\hline$\Omega_{b} h^{2}$ & 0.0222 & 0.0008 \\
$\Omega_{c} h^{2}$ & 0.1197 & 0.0030 \\
$10^{2} \theta_{A}$ & 1.0409 & 0.0050 \\
$10^{9} A_{s}$ & 2.196 & 0.1 \\
$n_{s}$ & 0.9655 & 0.010 \\
$\tau$ & 0.078 & 0.020 \\
$\Sigma m_{\nu}(\mathrm{meV})$ & 60 & 20 \\
$\Omega_{k}$ & 0 & 0.01 \\
$w_{0}$ & -1 & 0.3 \\
$w_{a}$ & 0 & 0.6 \\
$N_{\mathrm{eff}}$ & 3.046 & 0.080 \\
$\Omega_{e}$ & 0.007 & 0.002 \\
\hline \hline
\end{tabular}

$$
\hat{C}_{\ell}^{X Y}=\frac{1}{2 \ell+1} \sum_{m=-\ell}^{m=\ell} x_{\ell m}^{*} y_{\ell m} .
$$

This formula omits beam-smoothing effects and the subtraction of a noise term, which we account for below. The estimated power spectrum is a sum of many random variables of finite variance, and to good approximation follows a Gaussian distribution. This approximation breaks down at large scales but does not have a significant impact on expected errors. For a full-sky survey, we have

$$
\begin{aligned}
-2 \ln \mathcal{L}(\boldsymbol{\theta})= & -2 \sum_{\ell} \ln p\left(\hat{C}_{\ell} \mid \boldsymbol{\theta}\right) \\
= & \sum_{\ell}\left[\left(\hat{C}_{\ell}-C_{\ell}(\boldsymbol{\theta})\right)^{\top} \mathbb{C}_{\ell}^{-1}(\boldsymbol{\theta})\left(\hat{C}_{\ell}-C_{\ell}(\boldsymbol{\theta})\right)\right. \\
& \left.+\ln \operatorname{det}\left(2 \pi \mathbb{C}_{\ell}(\boldsymbol{\theta})\right)\right]
\end{aligned}
$$

where $\hat{C}_{\ell}=\left(\hat{C}_{\ell}^{T T}, \hat{C}_{\ell}^{T E}, \ldots\right)$ contains auto- and crossspectra and $\mathbb{C}_{\ell}$ is their covariance matrix. Inserting this likelihood into Eq. (6) and neglecting parameter dependence in the power spectrum covariance matrix, one obtains

$$
F_{i j}=\sum_{\ell} \frac{\partial C_{l}^{\top}}{\partial \theta_{i}} \mathbb{C}_{\ell}^{-1} \frac{\partial C_{l}}{\partial \theta_{j}}
$$

From Eq. (A1), and applying Wick's theorem, the covariance matrix for the power spectra has elements

$$
\begin{aligned}
\mathbb{C}\left(\hat{C}_{l}^{\alpha \beta}, \hat{C}_{l}^{\gamma \delta}\right)= & \frac{1}{(2 l+1) f_{\text {sky }}}\left[\left(C_{l}^{\alpha \gamma}+N_{l}^{\alpha \gamma}\right)\left(C_{l}^{\beta \delta}+N_{l}^{\beta \delta}\right)\right. \\
& \left.+\left(C_{l}^{\alpha \delta}+N_{l}^{\alpha \delta}\right)\left(C_{l}^{\beta \gamma}+N_{l}^{\beta \gamma}\right)\right],
\end{aligned}
$$

where $\alpha, \beta, \gamma, \delta \in\left\{T, E, B, \kappa_{c}\right\}$, and $f_{\text {sky }}$ accounts for the loss of information due to partial sky coverage [87,88]. Noise spectra are generated for each observable given input noise properties such as CMB map sensitivities. We assume additive white noise for the $\mathrm{CMB}$,

TABLE IV. Specification for the Planck and WMAP experiments used in the analysis, assuming white-noise properties. We define Planck-2015 to reproduce the constraints from Planck 2015 data; for Planck-pol we use the Planck Blue Book scaling factors to convert to polarization. For WMAP-pol we recover an optical depth uncertainty that matches the WMAP9 data. When combining with S3 and $\mathrm{S} 4$, we include Planck-pol data across the full $f_{\text {sky }}=0.44$ at large scales $\left(\ell<\ell_{\text {min }}^{\mathrm{S} 3 / \mathrm{S} 4}\right)$ and across $f_{\text {sky }}=0.2$ in the multipole range $\ell_{\text {min }}^{\mathrm{S} 3 / \mathrm{S} 4}<\ell<2500$, as the useful Planck data and S3/S4 will likely not overlap completely. Our results are insensitive to the exact choice of this nonoverlapping region size. When using WMAP-pol data we substitute it in at large scales $\left(\ell<\ell_{\text {min }}^{\mathrm{S} 3 / \mathrm{S} 4}\right)$ over $f_{\text {sky }}=0.74$.

\begin{tabular}{lccccccc}
\hline \hline Experiment & $f_{\text {sky }}$ & $\nu / \mathrm{GHz}$ & $l_{\min }$ & $l_{\max }$ & FWHM $/ \operatorname{arcmin}$ & $\Delta T / \mu \mathrm{K}-\operatorname{arcmin}$ & $\Delta P / \mu \mathrm{K}-\operatorname{arcmin}$ \\
\hline Planck-2015 & 0.44 & $\begin{array}{c}30,44,70,100, \\
143,217,353\end{array}$ & 2 & 2500 & $33,23,14,10,7$, & $145,149,137$, & $\cdots, \cdots, 450, \cdots, \cdots, \cdots, \cdots$ \\
Planck-pol & 0.44 & & & $5,5,5$ & $65,43,66,200$ & $\cdots, \cdots, 450,103,81$, \\
WMAP-pol & 0.74 & $33,41,64,94$ & 2 & 1000 & $41,28,21,13$ & $\cdots, \cdots, 298,296$ & $425,420,424, \cdots$ \\
\hline \hline
\end{tabular}


TABLE V. Specification for current BAO-15 data (top) and forecast DESI data (bottom). We derive the expected fractional uncertainties on $r_{s} / d_{V}$ for DESI from the fractional errors on $D_{A} / r_{s}$ and $H(z)$ forecast in [25]. Using the $r_{s} / d_{V}$ observable discards information in redshift-space distortions of the galaxy correlation function by treating the clustering as isotropic. The absolute values correspond to a $\Lambda \mathrm{CDM}$ model with $\Sigma m_{\nu}=$ $60 \mathrm{meV}$.

\begin{tabular}{lcclc}
\hline \hline Experiment & Redshift & $\frac{\sigma\left(r_{s} / d_{V}\right)}{\left(r_{s} / d_{V}\right)}(\%)$ & $\sigma\left(r_{s} / d_{V}\right)$ & References \\
\hline 6dFGRS & 0.106 & 4.83 & 0.0084 & {$[50]$} \\
SDSS MGS & 0.15 & 3.87 & 0.015 & {$[51]$} \\
LowZ & 0.32 & 2.35 & 0.0023 & {$[20]$} \\
C-MASS & 0.57 & 1.33 & 0.00071 & {$[20]$} \\
DESI & 0.15 & 1.89 & 0.0041 & {$[25]$} \\
& 0.25 & 1.26 & 0.0017 & \\
& 0.35 & 0.98 & 0.00088 & \\
& 0.45 & 0.80 & 0.00055 & \\
& 0.55 & 0.68 & 0.00038 & \\
& 0.65 & 0.60 & 0.00028 & \\
& 0.75 & 0.52 & 0.00021 & \\
& 0.85 & 0.51 & 0.00018 & \\
& 0.95 & 0.56 & 0.00018 & \\
& 1.05 & 0.59 & 0.00017 & \\
& 1.15 & 0.60 & 0.00016 & \\
& 1.25 & 0.57 & 0.00014 & \\
& 1.35 & 0.66 & 0.00015 & \\
& 1.45 & 0.75 & 0.00016 & \\
& 1.55 & 0.95 & 0.00019 & \\
& 1.65 & 1.48 & 0.00028 & \\
& 1.75 & 2.28 & 0.00041 & \\
& 1.85 & 3.03 & 0.00052 & \\
\hline \hline
\end{tabular}

$$
N_{\ell}^{\alpha \alpha}=(\Delta T)^{2} \exp \left(\frac{\ell(\ell+1) \theta_{\mathrm{FWHM}}^{2}}{8 \ln 2}\right),
$$

for $\alpha \in\{T, E, B\}$, where $\Delta T$ ( $\Delta P$ for polarization) is the map sensitivity in $\mu \mathrm{K}$-arcmin and $\theta_{\mathrm{FWHM}}$ is the beam width. This is an optimistic approximation: Real noise-spectra from ground-based experiments have a dominant contribution from atmospheric variance at large scales (see, e.g., Fig. 4 of Das et al. [82]). The atmosphere is weakly polarized, and hence the white-noise approximation is better in $E$ and $B$ than $T$. The CMB lensing reconstruction noise is calculated using the quadratic-estimator formalism [7]. As described in the main text, we neglect non-Gaussian terms in the power spectrum covariance, and we also neglect the $B B$ spectrum as it does not contribute significantly to upcoming constraints and has a highly nonGaussian covariance [40].
We add information from BAO experiments by computing the BAO Fisher matrix,

$$
F_{i j}^{\mathrm{BAO}}=\sum_{k} \frac{1}{\sigma_{f, k}^{2}} \frac{\partial f_{k}}{\partial \theta_{i}} \frac{\partial f_{k}}{\partial \theta_{j}},
$$

where $f_{k} \equiv f\left(z_{k}\right)=r_{s} / d_{V}\left(z_{k}\right)$ is the sound horizon at photon-baryon decoupling $r_{s}$ over the volume distance $d_{V}$ to the source galaxies at redshift $z_{k}$. These real and forecast data are reported in Table $V$.

The total Fisher information matrix is given by the sum of the $\mathrm{CMB}$ and $\mathrm{BAO}$ Fisher matrices, and is inverted to forecast parameter covariances. An alternative Markov Chain Monte Carlo (MCMC) approach using simulated data can be taken to account for non-Gaussianity of the posterior (e.g., [29]), but the Gaussian approximation is likely increasingly good as the data quality improve from Planck through S3 to S4.

Our forecasting code, OXFISH, has been developed for this analysis and is used to forecast parameter covariance matrices in one coherent PYTHON package. The code interfaces with the CAMB code for evaluation of power spectra. We compare with real data or previous work where possible. We construct the Planck-2015 (P15) specification, given in Table IV, to produce constraints that match the $\Lambda \mathrm{CDM}$ uncertainties from the Planck Collaboration [21], with the beam sizes and noise levels matching the detector sensitivities in [89].

We also forecast the neutrino mass constraint from $\mathrm{P} 15+\mathrm{BAO}$, finding $\sigma\left(\Sigma m_{\nu}\right)=103 \mathrm{meV}$. Placing the peak of the posterior at the fiducial $\Sigma m_{\nu}=60 \mathrm{meV}$, this corresponds to $\Sigma m_{\nu}<245 \mathrm{meV}$ at $95 \%$ confidence, comparable to the actual result of $\Sigma m_{\nu}<230 \mathrm{meV}$ [21]. This includes joint light-curve supernovae (JLA SNe) data and an $H_{0}$ prior, but these are expected to have a small impact. We also agree with [30-32] on the neutrino mass constraint for the $\mathrm{S} 4+\mathrm{DESI}$ data combination, finding $\sigma\left(\Sigma m_{\nu}\right)=$ $15 \mathrm{meV}$ if we assume that the reionization bump is measured.

We use the following parameters for the curves in Fig. 10: Solid curve, $\left\{\Omega_{b} h^{2}=0.0222, \Omega_{c} h^{2}=0.0120\right.$, $\Sigma m_{\nu}=3 \mathrm{meV}, \tau=0.067,10^{2} \theta_{A}=1.0417,10^{9} A_{s}=$ 2.15, $\left.n_{s}=0.9647, \sigma_{8}=0.835, H_{0}=68.0\right\}$; dashed curve, $\left\{\Omega_{b} h^{2}=0.0222, \Omega_{c} h^{2}=0.0119, \Sigma m_{\nu}=117 \mathrm{meV}, \tau=\right.$ $0.089,10^{2} \theta_{A}=1.0399,10^{9} A_{s}=2.24, n_{s}=0.9663, \sigma_{8}=0.822$, $\left.H_{0}=66.6\right\}$. 
[1] M. C. Gonzalez-Garcia and Y. Nir, Rev. Mod. Phys. 75, 345 (2003).

[2] M. Maltoni, T. Schwetz, M. Tórtola, and J. W. F. Valle, New J. Phys. 6, 122 (2004).

[3] A. Y. Smirnov, J. Phys. Conf. Ser. 53, 44 (2006).

[4] G. J. Feldman, J. Hartnell, and T. Kobayashi, Adv. High Energy Phys. 2013, 1 (2013).

[5] A. G. Doroshkevich, Y. B. Zeldovich, R. A. Syunyaev, and M. Y. Khlopov, Pisma Astron. Zh. 6, 457 (1980).

[6] A. G. Doroshkevich, M. I. Khlopov, R. A. Sunyaev, A. S. Szalay, and I. B. Zeldovich, Ann. N.Y. Acad. Sci. 375, 32 (1981).

[7] W. Hu and S. Dodelson, Annu. Rev. Astron. Astrophys. 40, 171 (2002).

[8] A. D. Dolgov, Phys. Rep. 370, 333 (2002).

[9] Ø. Elgarøy and O. Lahav, New J. Phys. 7, 61 (2005).

[10] M. Tegmark, Phys. Scr. T T121, 153 (2005).

[11] S. Hannestad, Phys. Rev. Lett. 95, 221301 (2005).

[12] K. Ichikawa, M. Fukugita, and M. Kawasaki, Phys. Rev. D 71, 043001 (2005).

[13] J. Lesgourgues and S. Pastor, Phys. Rep. 429, 307 (2006).

[14] M. Fukugita, Nucl. Phys. B, Proc. Suppl. 155, 10 (2006).

[15] C. Giunti and C. W. Kim, Fundamentals of Neutrino Physics and Astrophysics (Oxford University Press, Oxford, United Kingdom, 2007).

[16] F. B. Abdalla and S. Rawlings, Mon. Not. R. Astron. Soc. 381, 1313 (2007).

[17] E. Komatsu et al. (WMAP Collaboration), Astrophys. J. Suppl. Ser. 180, 330 (2009).

[18] Measurements of Neutrino Mass, Proceedings of the International School of Physics "Enrico Fermi," Course No. CLXX, edited by F. Ferroni, F. Vissani, and C. Brofferio (IOS Press, Amsterdam, 2009).

[19] S. Hannestad, Prog. Part. Nucl. Phys. 65, 185 (2010).

[20] L. Anderson, É. Aubourg, S. Bailey, F. Beutler, V. Bhardwaj, M. Blanton, A. S. Bolton, J. Brinkmann, J. R. Brownstein, A. Burden et al., Mon. Not. R. Astron. Soc. 441, 24 (2014).

[21] P. A. R. Ade, N. Aghanim, M. Arnaud, M. Ashdown, J. Aumont, C. Baccigalupi, A. J. Banday, R. B. Barreiro, J. G. Bartlett et al. (Planck Collaboration), arXiv:1502.01589.

[22] N. Palanque-Delabrouille, C. Yèche, A. Borde, J.-M. Le Goff, G. Rossi, M. Viel, É. Aubourg, S. Bailey, J. Bautista, M. Blomqvist et al., Astron. Astrophys. 559, A85 (2013).

[23] N. Palanque-Delabrouille, C. Yeche, J. Baur, C. Magneville, G. Rossi, J. Lesgourgues, A. Borde, E. Burtin, J.-M. LeGoff, J. Rich et al., J. Cosmol. Astropart. Phys. 11 (2015) 011.

[24] T. D. Kitching, A. F. Heavens, L. Verde, P. Serra, and A. Melchiorri, Phys. Rev. D 77, 103008 (2008).

[25] A. Font-Ribera, P. McDonald, N. Mostek, B. A. Reid, H.-J. Seo, and A. Slosar, J. Cosmol. Astropart. Phys. 05 (2014) 023.

[26] F. Villaescusa-Navarro, P. Bull, and M. Viel, Astrophys. J. 814, 146 (2015).

[27] E.-M. Mueller, F. de Bernardis, R. Bean, and M. D. Niemack, Phys. Rev. D 92, 063501 (2015).

[28] J. Errard, S. M. Feeney, H. V. Peiris, and A. H. Jaffe, arXiv: 1509.06770.

[29] A. C. Hall and A. Challinor, Mon. Not. R. Astron. Soc. 425, 1170 (2012).
[30] K. N. Abazajian, K. Arnold, J. Austermann, B. A. Benson, C. Bischoff, J. Bock, J. R. Bond, J. Borrill, E. Calabrese, J. E. Carlstrom et al., Astropart. Phys. 63, 66 (2015).

[31] W. L. K. Wu, J. Errard, C. Dvorkin, C. L. Kuo, A. T. Lee, P. McDonald, A. Slosar, and O. Zahn, Astrophys. J. 788, 138 (2014).

[32] Z. Pan and L. Knox, arXiv:1506.07493 [Mon. Not. R. Astron. Soc. (to be published)].

[33] Z. Hou, C. L. Reichardt, K. T. Story, B. Follin, R. Keisler, K. A. Aird, B. A. Benson, L. E. Bleem, J. E. Carlstrom, C. L. Chang et al., Astrophys. J. 782, 74 (2014).

[34] E. Komatsu, K. M. Smith, J. Dunkley, C. L. Bennett, B. Gold, G. Hinshaw, N. Jarosik, D. Larson, M. R. Nolta, L. Page et al., Astrophys. J. Suppl. Ser. 192, 18 (2011).

[35] W. Hu, D. J. Eisenstein, and M. Tegmark, Phys. Rev. Lett. 80, 5255 (1998).

[36] S. Bird, M. Viel, and M. G. Haehnelt, Mon. Not. R. Astron. Soc. 420, 2551 (2012).

[37] R. Mandelbaum, J. Instrum. 10, C05017 (2015).

[38] A. Lewis and A. Challinor, Phys. Rep. 429, 1 (2006).

[39] S. Das, B. D. Sherwin, P. Aguirre, J. W. Appel, J. R. Bond, C. S. Carvalho, M. J. Devlin, J. Dunkley, R. Dünner, T. Essinger-Hileman et al., Phys. Rev. Lett. 107, 021301 (2011).

[40] A. Benoit-Lévy, K. M. Smith, and W. Hu, Phys. Rev. D 86, 123008 (2012).

[41] D. J. Eisenstein et al. (SDSS Collaboration), Astrophys. J. 633, 560 (2005).

[42] N. Aghanim, M. Arnaud, M. Ashdown, J. Aumont, C. Baccigalupi, A. J. Banday, R. B. Barreiro, J. G. Bartlett, N. Bartolo et al. (Planck Collaboration), arXiv:1507.02704.

[43] S. Naess, M. Hasselfield, J. McMahon, M. D. Niemack, G. E. Addison, P. A. R. Ade, R. Allison, M. Amiri, N. Battaglia, J. A. Beall et al., J. Cosmol. Astropart. Phys. 10 (2014) 007.

[44] P. A. R. Ade, Y. Akiba, A. E. Anthony, K. Arnold, M. Atlas, D. Barron, D. Boettger, J. Borrill, S. Chapman, Y. Chinone et al. (Polarbear Collaboration), Astrophys. J. 794, 171 (2014).

[45] R. Keisler, S. Hoover, N. Harrington, J. W. Henning, P. A. R. Ade, K. A. Aird, J. E. Austermann, J. A. Beall, A. N. Bender, B. A. Benson et al., Astrophys. J. 807, 151 (2015).

[46] E. Calabrese, R. Hložek, N. Battaglia, J. R. Bond, F. de Bernardis, M. J. Devlin, A. Hajian, S. Henderson, J. C. Hil, A. Kosowsky et al., J. Cosmol. Astropart. Phys. 08 (2014) 010.

[47] B. A. Benson, P. A. R. Ade, Z. Ahmed, S. W. Allen, K. Arnold, J. E. Austermann, A. N. Bender, L. E. Bleem, J. E. Carlstrom, C. L. Chang et al., Proc. SPIE Int. Soc. Opt. Eng. 9153, 91531P (2014).

[48] T. Matsumura, Y. Akiba, J. Borrill, Y. Chinone, M. Dobbs, H. Fuke, A. Ghribi, M. Hasegawa, K. Hattori, M. Hattori et al., J. Low Temp. Phys. 176, 733 (2014).

[49] A. Kogut, D. J. Fixsen, D. T. Chuss, J. Dotson, E. Dwek, M. Halpern, G. F. Hinshaw, S. M. Meyer, S. H. Moseley, M. D. Seiffert et al., J. Cosmol. Astropart. Phys. 07 (2011) 025.

[50] F. Beutler, C. Blake, M. Colless, D. H. Jones, L. StaveleySmith, L. Campbell, Q. Parker, W. Saunders, and F. Watson, Mon. Not. R. Astron. Soc. 416, 3017 (2011). 
[51] A. J. Ross, L. Samushia, C. Howlett, W. J. Percival, A. Burden, and M. Manera, Mon. Not. R. Astron. Soc. 449, 835 (2015).

[52] J. Comparat, T. Delubac, S. Jouvel, A. Raichoor, J. Kneib, C. Yeche, F. B. Abdalla, C. Le Cras, C. Maraston, D. M. Wilkinson et al., arXiv:1509.05045.

[53] M. Levi, C. Bebek, T. Beers, R. Blum, R. Cahn, D. Eisenstein, B. Flaugher, K. Honscheid, R. Kron, O. Lahav et al., arXiv:1308.0847.

[54] E. Anderes, B. D. Wandelt, and G. Lavaux, Astrophys. J. 808, 152 (2015).

[55] M. M. Schmittfull, A. Challinor, D. Hanson, and A. Lewis, Phys. Rev. D 88, 063012 (2013).

[56] A. Lewis, A. Challinor, and A. Lasenby, Astrophys. J. 538, 473 (2000).

[57] Planck Collaboration, The Scientific Programme of Planck (Bluebook), (ESA-SCI(2005)1, 2005).

[58] W. Hu and T. Okamoto, Astrophys. J. 574, 566 (2002).

[59] K. M. Smith, D. Hanson, M. LoVerde, C. M. Hirata, and O. Zahn, J. Cosmol. Astropart. Phys. 06 (2012) 014.

[60] T. Namikawa, S. Saito, and A. Taruya, J. Cosmol. Astropart. Phys. 12 (2010) 027.

[61] Z. Pan, L. Knox, and M. White, Mon. Not. R. Astron. Soc. 445, 2941 (2014).

[62] C. M. Hirata and U. Seljak, Phys. Rev. D 68, 083002 (2003).

[63] E. Calabrese, A. Slosar, A. Melchiorri, G. F. Smoot, and O. Zahn, Phys. Rev. D 77, 123531 (2008).

[64] Planck Collaboration, Astron. Astrophys. 571, A16 (2014).

[65] K. M. Smith, W. Hu, and M. Kaplinghat, Phys. Rev. D 74, 123002 (2006).

[66] G. Hinshaw, D. Larson, E. Komatsu, D. N. Spergel, C. L. Bennett, J. Dunkley, M. R. Nolta, M. Halpern, R. S. Hill, N. Odegard et al., Astrophys. J. Suppl. Ser. 208, 19 (2013).

[67] L. Koopmans, J. Pritchard, G. Mellema, J. Aguirre, K. Ahn, R. Barkana, I. van Bemmel, G. Bernardi, A. Bonaldi, F. Briggs et al., Proc. Sci., AASKA14 (2015) 001.

[68] A. Liu, J. R. Pritchard, R. Allison, A. R. Parsons, U. Seljak, and B. D. Sherwin, arXiv:1509.08463.

[69] Planck Collaboration, arXiv:1502.01591.

[70] J. Hamann, S. Hannestad, and Y. Y. Y. Wong, J. Cosmol. Astropart. Phys. 11 (2012) 052.
[71] M. Chevallier and D. Polarski, Int. J. Mod. Phys. D 10, 213 (2001).

[72] E. V. Linder, Phys. Rev. Lett. 90, 091301 (2003).

[73] M. Doran and G. Robbers, J. Cosmol. Astropart. Phys. 6 (2006) 026.

[74] E. Calabrese, R. de Putter, D. Huterer, E. V. Linder, and A. Melchiorri, Phys. Rev. D 83, 023011 (2011).

[75] S. Joudaki and M. Kaplinghat, Phys. Rev. D 86, 023526 (2012).

[76] W. Fang, W. Hu, and A. Lewis, Phys. Rev. D 78, 087303 (2008).

[77] R. Trotta, Mon. Not. R. Astron. Soc. 378, 72 (2007).

[78] J. R. Bond, G. Efstathiou, and M. Tegmark, Mon. Not. R. Astron. Soc. 291, L33 (1997)

[79] B. D. Sherwin, J. Dunkley, S. Das, J. W. Appel, J. R. Bond, C. S. Carvalho, M. J. Devlin, R. Dünner, T. EssingerHileman, J. W. Fowler et al., Phys. Rev. Lett. 107, 021302 (2011).

[80] S. Hannestad, Annu. Rev. Nucl. Part. Sci. 56, 137 (2006).

[81] P. A. R. Ade, N. Aghanim, M. Arnaud, M. Ashdown, J. Aumont, C. Baccigalupi, A. J. Banday, R. B. Barreiro, N. Bartolo et al. (Planck Collaboration), arXiv:1502.01590.

[82] S. Das, T. Louis, M. R. Nolta, G. E. Addison, E. S. Battistelli, J. Bond, E. Calabrese, D. C. M. J. Devlin, S. Dicker, J. Dunkley et al., J. Cosmol. Astropart. Phys. 04 (2014) 014.

[83] P. A. Abell, J. Allison, S. F. Anderson, J. R. Andrew, J. R. P. Angel, L. Armus, D. Arnett, S. J. Asztalos, T. S. Axelrod et al. (LSST Science Collaboration), arXiv:0912.0201.

[84] C. Carbone, C. Fedeli, L. Moscardini, and A. Cimatti, J. Cosmol. Astropart. Phys. 03 (2012) 023.

[85] M. Costanzi Alunno Cerbolini, B. Sartoris, J.-Q. Xia, A. Biviano, S. Borgani, and M. Viel, J. Cosmol. Astropart. Phys. 06 (2013) 020.

[86] D. J. E. Marsh, E. Macaulay, M. Trebitsch, and P. G. Ferreira, Phys. Rev. D 85, 103514 (2012).

[87] M. P. Hobson and J. Magueijo, Mon. Not. R. Astron. Soc. 283, 1133 (1996).

[88] R. de Putter, O. Zahn, and E. V. Linder, Phys. Rev. D 79, 065033 (2009).

[89] R. Adam, P. A. R. Ade, N. Aghanim, Y. Akrami, M. I. R. Alves, M. Arnaud, F. Arroja, J. Aumont, C. Baccigalupi et al. (Planck Collaboration), arXiv:1502.01582. 